Pacific

Journal of

Mathematics

\title{
EFFICIENT FUNDAMENTAL CYCLES OF CUSPED
} HYPERBOLIC MANIFOLDS

Thilo Kuessner 


\title{
EFFICIENT FUNDAMENTAL CYCLES OF CUSPED HYPERBOLIC MANIFOLDS
}

\author{
Thilo Kuessner
}

Let $M$ be a manifold (with boundary) of dimension $\geq 3$, such that its interior admits a hyperbolic metric of finite volume. We discuss the possible limits arising from sequences of relative fundamental cycles approximating the simplicial volume $\|M, \partial M\|$, using ergodic theory of unipotent actions. As applications, we extend results of Jungreis and Calegari from closed hyperbolic to finite-volume hyperbolic manifolds:

a) Strict subadditivity of simplicial volume with respect to isometric glueing along geodesic surfaces, and

b) nontriviality of the foliated Gromov norm for "most" foliations with two-sided branching.

\section{Introduction.}

Gromov defined the simplicial volume $\|M, \partial M\|$ of a manifold $M$ as the "minimal cardinality of a triangulation with real coefficients". That means, for an $n$-dimensional compact, connected, orientable manifold $M$ with (possibly empty) boundary $\partial M$, define

$$
\|M, \partial M\|:=\inf \left\{\sum_{i=1}^{r}\left|a_{i}\right|: \sum_{i=1}^{r} a_{i} \sigma_{i} \text { represents }[M, \partial M]\right\} .
$$

Here, $[M, \partial M] \in H_{n}(M, \partial M ; \mathbb{R})$ is the image of a generator of $H_{n}(M, \partial M$; $\mathbb{Z}) \simeq \mathbb{Z}$ under the canonical homomorphism $H_{n}(M, \partial M ; \mathbb{Z}) \rightarrow H_{n}(M, \partial M ;$ $\mathbb{R})$.

The simplicial volume quantifies the topological complexity of a manifold. It is nontrivial if $\operatorname{int}(M)$ admits a complete metric of sectional curvature $\leq-a^{2}$ and finite volume. In particular the Gromov-Thurston theorem ([9], [19]) states for finite-volume hyperbolic manifolds $\|M, \partial M\|=\frac{1}{V_{n}} \operatorname{Vol}(M)$, where $V_{n}$ is the volume of a regular ideal simplex in $\mathbb{H}^{n}$. This exhibits hyperbolic volume as a homotopy invariant, complementing the Chern-GaußBonnet theorem, which implies homotopy invariance of hyperbolic volume for even-dimensional manifolds. Homotopy invariance of hyperbolic volume was used by Gromov to give a more topological proof of Mostow's rigidity theorem. In the meantime, various more general rigidity theorems have been proved, again using the simplicial volume. 
On a finite-volume hyperbolic manifold, there does not exist a fundamental cycle actually having $l^{1}$-norm $\frac{1}{V_{n}} \operatorname{Vol}(M)$. However, there is a measure cycle, supported on the set of regular ideal simplices, the so-called smearing of a regular ideal simplex, having this norm: After identifying the set of (ordered) regular ideal simplices with $\operatorname{Isom}\left(\mathbb{H}^{n}\right)=\operatorname{Isom}^{+}\left(\mathbb{H}^{n}\right) \cup \operatorname{Isom}^{-}\left(\mathbb{H}^{n}\right)$, it is the signed measure $\frac{1}{2 V_{n}}\left(\mathrm{Haar}-r^{*} \mathrm{Haar}\right)$, where Haar is the Haar measure on $\operatorname{Isom}^{+}\left(\mathbb{H}^{n}\right)$ and $r$ is an orientation-reversing isometry. This measure cycle can be approximated by authentic singular chains, i.e., finite linear combinations of (nonideal) simplices (and this proves the Gromov-Thurston theorem, cf. [2] for details of the proof in the case of closed manifolds).

Technically, the main part of this paper is devoted to the question of to which extent this construction is unique, i.e., whether there exist sequences of fundamental cycles with $l^{1}$-norms converging to $\frac{1}{V_{n}} \operatorname{Vol}(M)$ which do not approximate Gromov's smearing construction.

For closed manifolds of dimension $\geq 3$, it was shown in [11] by Jungreis that any such sequence must converge to Gromov's smearing cycle. In this paper we extend this rigidity results to hyperbolic manifolds of finite volume which are either of dimension $\geq 4$ or which are of dimension 3 and not Gieseking-like (see Definition 4.4).

Moreover, we obtain restrictions on sequences of fundamental cycles with $l^{1}$-norms converging to $\frac{1}{V_{n}} \operatorname{Vol}(M)$ on (possibly Gieseking-like) finite-volume hyperbolic manifolds of dimension $\geq 3$, which allow to conclude: If $F$ is a closed geodesic hypersurface, then the limits of such sequences do invoke simplices intersecting $F$ 'transversally' (see Definition 5.1). This property can actually be restated as $\left\|M_{F}, \partial M_{F}\right\|>\|M, \partial M\|$, where $M_{F}$ is obtained by cutting $M$ along $F$.

As applications, we extend results of Jungreis and Calegari to hyperbolic manifolds with cusps.

Glueing along boundaries. Consider manifolds $M_{1}, M_{2}$, a homeomorphism $f: A_{1} \rightarrow A_{2}$ between subsets $A_{i} \subset \partial M_{i}$, and let $M=M_{1} \cup_{f} M_{2}$ be the glued manifold. In general, it is hard to compare $\|M\|$ to $\left\|M_{1}\right\|+\left\|M_{2}\right\|$. One can prove " $\geq$ " if the $A_{i}$ are incompressible and amenable, and even "=" if, in addition, they are connected components of $\partial M_{i}$, cf. [9] and [12].

Theorem 6.3. Let $n \geq 3$ and let $M_{1}, M_{2}$ be compact $n$-manifolds with boundaries $\partial M_{i}=\partial_{0} M_{i} \cup \partial_{1} M_{i}$, such that $M_{i}-\partial_{0} M_{i}$ admit incomplete hyperbolic metrics of finite volume with totally geodesic boundaries $\partial_{1} M_{i}$. If $\partial_{1} M_{i}$ are not empty, $f: \partial_{1} M_{1} \rightarrow \partial_{1} M_{2}$ is an isometry and $M=M_{1} \cup_{f} M_{2}$, then

$$
\|M, \partial M\|<\left\|M_{1}, \partial M_{1}\right\|+\left\|M_{2}, \partial M_{2}\right\| .
$$

The same statement holds if one glues only along some connected components of $\partial_{1} M_{i}$. One also has an analogous statement if two totally geodesic 
boundary components of the same hyperbolic manifold are glued by an isometry.

One point of interest in Theorem 6.3 is that it serves, in the case of 3manifolds, as a main step for a general glueing inequality. In [12], we prove:

Theorem. For a compact 3-manifold $M\|D M\|<2\|M, \partial M\|$ holds if and only if $\|\partial M\|>0$, i.e., if $\partial M$ consists not only of spheres and tori.

(Here, $D M$ is the manifold obtained by glueing two differently oriented copies of $M$ via the identity of $\partial M$. Note that $\|D M\| \leq 2\|M, \partial M\|$ trivially holds.) This theorem may be seen as a generalisation of Theorem 6.3, saying that any efficient fundamental cycle on a 3 -manifold with $\mathbb{Z}_{2}$-symmetry has to intersect the fixed point set 'transversally'. It is maybe worth pointing out that for the proof of this theorem in [12] we need to have Theorem 6.3 also for the case of cusps.

Another (direct) corollary from Theorem 6.3 and Mostow rigidity is that (under the assumptions of Theorem 6.3), in dimensions $\geq 4$, we get the same inequality for any homeomorphisms $f$. This theorem seems to be hardly available by topological methods. The analogous statement in dimension 3 was recently shown to be wrong by Soma ([18]). He proved: If $M_{1}, M_{2}$ are hyperbolic 3-manifolds of totally geodesic boundary and $f: \partial M_{1} \rightarrow \partial M_{2}$ is pseudo-Anosov, then $\lim _{n \rightarrow \infty}\left\|M_{1} \cup_{f^{n}} M_{2}\right\|=\infty$.

Foliated Gromov norm. The Gromov norm of a foliation/lamination $\mathcal{F}$ on a manifold $M$, as introduced in [4], is

$$
\begin{aligned}
\|M, \partial M\|_{\mathcal{F}}:=\inf \left\{\sum_{i=1}^{r}\left|a_{i}\right|: \sum_{i=1}^{r} a_{i} \sigma_{i}\right. \text { represents } & {[M, \partial M], } \\
& \left.\sigma_{i} \text { transverse to } \mathcal{F}\right\} .
\end{aligned}
$$

The difference $\|M, \partial M\|_{\mathcal{F}}-\|M, \partial M\|$ seems to quantify the amount of branching of the leaf space. Calegari proved:

- $\|M\|_{\mathcal{F}}=\|M\|$, when the leaf space is branched in at most one direction, and

- $\|M\|_{\mathcal{F}}>\|M\|$ for asymptotically separated laminations of closed hyperbolic manifolds of dimension $\geq 3$.

The first statement generalizes easily to manifolds with boundary. We extend the second statement as follows:

Theorem 7.5. Assume that the interior of $M$ is a hyperbolic n-manifold of finite volume. If $n \geq 3$ and $M$ is not Gieseking-like (Definition 4.4), and if $\mathcal{F}$ is an asymptotically separated lamination, then

$$
\|M, \partial M\|<\|M, \partial M\|_{\mathcal{F}} .
$$


We want to outline the content of this paper. In Chapter 3 we give a definition of "efficient fundamental chains", exhibit them as signed measures $\mu$ on the space of regular ideal simplices, show that they are absolute cycles (the boundary "escapes to infinity" for $\epsilon \rightarrow 0$ ), and derive ergodic decompositions of $\mu$ with respect to certain groups of reflections. Such (different) decompositions exist associated to all vertices of a fixed simplex $\Delta_{0}$. We show that the ergodic decomposition corresponding to the $i$-th vertex of $\Delta_{0}$ uses only the Haar measure and measures determined on the set of simplices having $i$-th vertex in a parabolic fixed point of $\Gamma$.

This is used in Chapter 5 to prove Theorem 5.3: If $F$ is a closed, totally geodesic hypersurface in a finite-volume hyperbolic manifold of dimension $\geq 3$ and $\mu$ is an efficient fundamental cycle, then $\mu\left(S_{F}\right)>0$, where $S_{F}$ is the set of simplices intersecting $F$ transversally. To give a rough explanation of the proof: The Haar measure does not vanish on $S_{F}$, hence $\mu\left(S_{F}\right)=0$ would imply that $\mu$ is determined on the set of simplices with all vertices in parabolic points, contradicting the fact that it must invoke simplices with faces in the cuspless hypersurface $F$.

In Chapter 6, Theorem 6.3 is derived from Theorem 5.3. Chapter 7 is devoted to the foliated Gromov norm and the proof of Theorem 7.5.

The simplicial volume of a nonorientable, disconnected manifold is the sum over the connected components of half of the simplicial volumina of the orientation coverings. We will give all proofs for connected, oriented manifolds, since all statements generalise directly. This includes that the orientations of glued manifolds are understood to fit together.

\section{Preliminaries.}

2.1. Volume of straight simplices. A simplex in hyperbolic space $\mathbb{H}^{n}$, with vertices $p_{0}, \ldots, p_{i}$, is called straight if it is the barycentric parametrization of the geodesic simplex with vertices $p_{0}, \ldots, p_{i}$.

Given two regular ideal (straight) $n$-simplices $\Delta_{0}$ and $\Delta$ in $\mathbb{H}^{n}$, with fixed orderings of their vertices, there is a unique $g \in \operatorname{Isom}\left(\mathbb{H}^{n}\right)$ mapping $\Delta_{0}$ to $\Delta$.

Hence, fixing a reference simplex $\Delta_{0}$, we have an $\operatorname{Isom}\left(\mathbb{H}^{n}\right)$-equivariant bijection between the set of ordered regular ideal $n$-simplices and Isom $\left(\mathbb{H}^{n}\right)$, this bijection being unique up to the choice of $\Delta_{0}$, i.e., up to multiplication with a fixed element of Isom $\left(\mathbb{H}^{n}\right)$.

As another consequence, all regular ideal $n$-simplices in $\mathbb{H}^{n}$ have the same volume, to be denoted $V_{n}$.

By [10], any straight $n$-simplex $\sigma$ in $\mathbb{H}^{n}$ satisfies $\operatorname{Vol}(\sigma) \leq V_{n}$ and equality is achieved only for regular ideal simplices.

2.2. Ergodic decomposition. For a topological space $X$, we consider Radon measures $\mu$ on $X$. This are, by definition, elements of $C_{c}^{*}(X)$, the 
dual of the space of compactly supported continuous functions. They have a decomposition $\mu=\mu^{+}-\mu^{-}$with $\mu^{+}, \mu^{-}$nonnegative Radon measures. (We will refer to $\mu$ as signed measure and to $\mu^{ \pm}$as measures.) A probability measure on $X$ is a measure $\mu$ with $\mu(X)=1$.

Let a group $G$ act on a topological space $X$. A probability measure $\mu$ is called ergodic if any $G$-invariant set has measure 0 or 1 . Denote by $\mathcal{E}$ the set of ergodic $G$-invariant probability measures on $X$.

Let $\mathcal{A}$ be the weak measure class induced by the measure class on $X$, i.e., the smallest $\sigma$-algebra $\mathcal{A}$ on $\mathcal{E}$ such that for all Borel sets $A \subset X$ the application $f_{A}: \mathcal{E} \rightarrow \mathbb{R}$ defined by

$$
f_{A}(\mu):=\mu(A)
$$

is measurable.

Lemma 2.1. Let a group $G$ act on a complete separable metric space $X$. If there exists a $G$-invariant probability measure on $X$, then the set $\mathcal{E}$ of ergodic $G$-invariant measures on $X$ is not empty and there is a decomposition map $\beta: X \rightarrow \mathcal{E}$.

Here, a decomposition map is a $G$-invariant map $\beta: X \rightarrow \mathcal{E}$, which is:

- Measurable with respect to $\mathcal{A}$,

- satisfies $e(\{x \in X: \beta(x)=e\})=1$ for all $e \in \mathcal{E}$, and

- for all $G$-invariant probability measures $\mu$ and Borel sets $A \subset X$ the following equality holds:

$$
\mu(A)=\int_{X} \beta(x)(A) d \mu(x) .
$$

For a proof of Lemma 2.1, see Theorem 4.2 in [21].

For later reference we state the following lemma, Part (i) of which is known as Alaoglu's theorem, whereas a proof of Part (ii) can be found in Lemma 3.2 of $[\mathbf{6}]$.

\section{Lemma 2.2.}

(i) Any weak-*-bounded sequence of signed Radon measures on a locally compact metric space has an accumulation point in the weak-*-topology.

(ii) If $\mu$ is the weak-*-limit of a sequence $\mu_{n}$ of measures on a space $X$, and $U \subset X$ is an open subset, then $\mu(U) \leq \liminf \mu_{n}(U)$.

Moreover, we recall that the support of a measure $\mu$ on $X$ is defined as the complement of the largest open set $U \subset X$ with the property $\mu(U)=0$.

2.3. Measure homology. The following explanations are not necessary (from a logical point of view) for our arguments, but may be helpful to understand the framework. For a topological space $X$, let $C^{0}\left(\Delta^{k}, X\right)$ be the space of singular $k$-simplices in $X$, topologized by the compact-opentopology. For a signed measure $\mu$ on $C^{0}\left(\Delta^{k}, X\right)$, one has its decomposition 
$\mu=\mu^{+}-\mu^{-}$as difference of two (nonnegative) Borel measures, and one defines its total variation as $\|\mu\|=\int d \mu^{+}+\int d \mu^{-}$.

Let $\mathcal{C}_{k}(X)$ be the vector space of all signed measures $\mu$ on $C^{0}\left(\Delta^{k}, X\right)$ which have compact support and finite total variation. (We assume finite total variation because we want $\|$.$\| to define a norm on \mathcal{C}_{k}(X)$. The condition 'compact support' is imposed because otherwise the map $j_{*}: H_{*}(X ; \mathbb{R}) \rightarrow$ $\mathcal{H}_{*}(X)$ defined below would, in general, not be surjective, see [19], 6.1.7 for examples of this phenomenon.) Let $\eta_{i}: \Delta^{k} \rightarrow \Delta^{k-1}$ be the $i$-th face map. It induces a map $\partial_{i}=\left(\eta_{i}^{*}\right)_{*}: \mathcal{C}_{k}(X) \rightarrow \mathcal{C}_{k-1}(X)$. We define the boundary operator $\partial:=\sum_{i=0}^{k} \partial_{i}$, to make $\mathcal{C}_{*}(X)$ a chain complex. We denote the homology groups of this chain complex by $\mathcal{H}_{*}(X)$.

We have an obvious inclusion $j: C_{*}(X) \rightarrow \mathcal{C}_{*}(X)$, where $C_{*}(X)$ are the singular chains, considered as finite linear combination of atomic measures. Clearly, $j$ is a chain map. Zastrow's Theorem 3.4. in [22] says that we get an isomorphism $j_{*}: H_{*}(M) \rightarrow \mathcal{H}_{*}(M)$ if $M$ is a smooth manifold (but not for arbitrary topological spaces $X)$.

The $l^{1}$-norm on $C_{*}(M)$ extends to the total variation $\|$.$\| on \mathcal{C}_{*}(M)$, and we get an induced pseudonorm on $\mathcal{H}_{*}(M)$. Thurston conjectured in [20] that the isomorphism $j_{*}$ should be an isometry for this pseudonorm. There seems not to exist a proof of this general conjecture so far, but if $M$ is a closed hyperbolic $n$-manifold, it follows easily from the identity $\|M\|=\frac{1}{V_{n}} \operatorname{Vol}(M)$ $([\mathbf{2}],[\mathbf{9}])$ that $j_{n}: H_{n}(M) \rightarrow \mathcal{H}_{n}(M)$ is an isometry.

\subsection{Intersection numbers.}

Definition 2.3. Let $M$ be an oriented differentiable $n$-manifold. For an immersed differentiable $n$-simplex $\sigma: \Delta^{n} \rightarrow M$, and $x \in M$, define

$$
\Phi_{x}(\sigma)=\sum_{y \in \sigma^{-1}(x)} \operatorname{sign} \operatorname{det} d \sigma(y) .
$$

For a singular chain $c=\sum_{j=1}^{r} a_{j} \sigma_{j}$, let $\Phi_{x}(c)=\sum_{j=1}^{r} a_{j} \Phi_{x}\left(\sigma_{j}\right)$.

Lemma 2.4. Let $M$ be a connected, oriented, smooth, noncompact n-manifold, $M^{\prime}$ an n-submanifold with boundary, such that $M-M^{\prime}$ is compact. Let $c=\sum_{j=1}^{r} a_{j} \sigma_{j}$ be a smooth singular $n$-chain representing the relative fundamental class $\left[M, M^{\prime}\right]$. Assume that all $\sigma_{j}$ are immersed smooth $n$ simplices. Then $\Phi_{x}(c)=1$ holds for almost all $x \in M-M^{\prime}$.

Proof. Let $K=\cup_{j=0}^{r} \mathrm{im}\left(\partial \sigma_{j}\right)$. $K$ is of measure zero, by Sard's lemma.

We want to show that $\Phi_{x}(c)$, as a function of $x$, is constant on $M-$ $\left(M^{\prime} \cup K\right)$. It is obvious that it is locally constant on $M-\left(M^{\prime} \cup K\right)$, since all $\sigma_{i}$ are immersed. It remains to prove: For all $x \in K \cap \operatorname{int}\left(M-M^{\prime}\right)$, there is a neighborhood $U$ of $x$ in $M$ such that $\Phi$. (c) is constant on $U \cap(M-K)$.

The point $x$ is contained in the image of finitely many $(n-1)$-simplices $\kappa_{1}, \ldots, \kappa_{k}$, which are boundary faces of some $\sigma_{i_{1}}, \ldots, \sigma_{i_{k}}$. (Note that the 
$\sigma_{i j}$ 's need not be distinct and that there might be further $\sigma_{i}$ 's containing $x$ in the interior of their image.) Since $\partial \sum_{j=1}^{r} a_{j} \sigma_{j}$ invokes only simplices whose image is contained in $M-M^{\prime}$, we necessarily have that all $\sigma_{i_{1}}, \ldots, \sigma_{i_{k}}$ cancel each other, i.e., there is a partition of $\left\{i_{1}, \ldots, i_{k}\right\}$ in some subsets, such that for each of these subsets of indices the sum of the corresponding coefficients $a_{i_{j}}$, multiplied with a sign according to orientation of $\sigma_{i_{j}}$, adds up to zero. This implies that $\Phi$. is constant in the intersection of a small neighborhood of $x$ with the complement of $K$ and, hence, also constant on all of $M-\left(M^{\prime} \cup K\right)$.

We now prove that this constant does not depend on the representative of the relative fundamental class. This implies that the constant must be 1 , since one can choose a triangulation as representative of the relative fundamental class.

If $c$ and $c^{\prime}$ are different representatives of $\left[M, M^{\prime}\right]$, we have that $c-c^{\prime}=$ $\partial w+t$ for some $w \in C_{n+1}(M)$ and $t \in C_{n}\left(M^{\prime}\right)$. Because $\partial w$ is a cycle, the same argument as above gives that $\Phi$. $(\partial w)$ is a.e. constant on all of $M$. This constant must be zero, since $\partial w$ has compact support in the noncompact manifold $M$. That means that $\Phi_{x}(c)-\Phi_{x}\left(c^{\prime}\right)=\Phi_{x}(t)$ for almost all $x \in M$. But $\Phi_{x}(t)=0$ for all $x \in \operatorname{int}\left(M-M^{\prime}\right)$.

2.5. Convergence of fundamental cycles. A major point of the next chapter will be to consider limiting objects of sequences of relative fundamental cycles of a finite-volume hyperbolic manifold $M$ with $l^{1}$-norms converging to the simplicial volume. Since straight simplices have volume smaller than $V_{n}$, there do not exist relative fundamental cycles actually having $l^{1}$-norm equal to $\frac{1}{V_{n}} \operatorname{Vol}(M)$. Hence, the limits of such sequences can not be just singular chains. What we are going to do is to embed the singular chain complex into a larger space, where any bounded sequence has accumulation points. A straightforward idea would be to use the inclusion $j: C_{n}(M) \rightarrow \mathcal{C}_{n}(M)$ and to consider weak-* accumulation points in $\mathcal{C}_{n}(M)$. This works perfectly well, however it is easy to see that the weak-* limits are just trivial measures. The reason is roughly the following: A singular chain with $l^{1}$-norm close to $\frac{1}{V_{n}} \operatorname{Vol}(M)$ has to have a very large part of its mass on simplices $\sigma$ with vol $(\operatorname{str}(\sigma))$ quite close to $V_{n}$. If we consider a compact set of simplices, it will have some upper bound (better than $V_{n}$ ) on $\operatorname{vol}(\operatorname{str}($.$) ).$ Hence, it will contribute very little to an almost efficient fundamental cycle, and the limiting measure will actually vanish on this set of simplices.

Therefore, to get nontrivial accumulation points, we are obliged to consider the larger space of simplices which might be ideal, i.e., whose lifts to $\mathbb{H}^{n}$ might have vertices in $\partial_{\infty} \mathbb{H}^{n}$. This, however, raises another problem: The space of ideal simplices in $M=\Gamma \backslash \mathbb{H}^{n}$ is not Hausdorff, and there is no theorem guaranteeing existence of weak-* accumulation points for signed measures on non-Hausdorff spaces. 
(If $M=\Gamma \backslash \mathbb{H}^{n}$ has finite volume, then the action of $\Gamma$ on $\partial_{\infty} \mathbb{H}^{n}$ has dense orbits. Thus the quotient $\Gamma \backslash S S_{i}(M)$ can not be Hausdorff as long as $S S_{i}(M)$ contains degenerate simplices. We will show in Section 2.5.3, however, that the action of $\Gamma$ on the subspace of nondegenerate $n$-simplices is properly discontinuous, i.e., after throwing away the degenerate simplices we get a Hausdorff quotient. A similar idea seems to have been exploited in the proof of Lemma 2.2 in [11] where the author restricted to a compact subset of $S S_{n}(M)$, i.e., to simplices with a lower volume bound.)

2.5.1. Straightening alternating chains. The symmetric group $S_{n+1}$ acts on the standard $n$-simplex $\Delta^{n}$ : Any permutation $\pi$ of vertices can be realised by a unique affine map $f_{\pi}: \Delta^{n} \rightarrow \Delta^{n}$. For a singular simplex $\sigma: \Delta^{n} \rightarrow M$ let alt $(\sigma):=\sum_{\pi \in S_{n+1}} \operatorname{sgn}(\pi) \sigma f_{\pi}$, and for a singular chain $c=\sum_{j=1}^{r} a_{j} \sigma_{j}$ define alt $(c):=\frac{1}{(n+1) !} \sum_{j=1}^{r} a_{j}$ alt $\left(\sigma_{j}\right)$. Clearly, $\|$ alt $(c)\|\leq\| c \|$.

For a simplex $\sigma$ in $\mathbb{H}^{n}$, we denote by $\operatorname{Str}(\sigma)$ the straight simplex with the same vertices as $\sigma$ (as in Section 2.1). A straight simplex in a hyperbolic manifold $M=\Gamma \backslash \mathbb{H}^{n}$ is the image of a straight simplex in $\mathbb{H}^{n}$ under the projection $p: \mathbb{H}^{n} \rightarrow \Gamma \backslash \mathbb{H}^{n}=M$. For a simplex $\sigma$ in $M$, its straightening $\operatorname{Str}(\sigma)$ is defined as $p(\operatorname{Str}(\widetilde{\sigma}))$, where $\widetilde{\sigma}$ is a simplex in $\mathbb{H}^{n}$ projecting to $\sigma$. Since straightening in $\mathbb{H}^{n}$ commutes with isometries, the definition of $\operatorname{Str}(\sigma)$ does not depend on the choice of $\widetilde{\sigma}$.

Finally, the straightening of a singular chain $\mathrm{c}=\sum_{j=1}^{r} a_{j} \sigma_{j}$ is defined as $\operatorname{Str}(c)=\sum_{j=1}^{r} a_{j} \operatorname{Str}\left(\sigma_{j}\right)$. Str $(c)$ is homologous to $c$, and clearly $\|\operatorname{Str}(c)\| \leq$ $\|c\|$ for any $c \in C_{*}(M)$. ( $\operatorname{Str}(c)$ may possibly have smaller norm than $c$, since different simplices can have the same straightenings.)

If $M^{\prime} \subset M$ is a convex subset (meaning that $\sigma \subset M^{\prime}$ implies $\operatorname{str}(\sigma) \subset M^{\prime}$ ), then $\operatorname{Str}: C_{*}\left(M, M^{\prime}\right) \rightarrow C_{*}\left(M, M^{\prime}\right)$ is well-defined.

2.5.2. Nondegenerate chains. Let $M$ be a hyperbolic manifold. We call a straight $i$-simplex $\sigma: \Delta^{i} \rightarrow N$ degenerate if two of its vertices are mapped to the same point, nondegenerate otherwise.

For $M^{\prime} \subset M$ a convex subset of $M$, we consider algvol : $C_{n}\left(M, M^{\prime}\right) \rightarrow$ $R$ which maps $\sigma \in C_{n}(M)$ to the algebraic volume (see [2], p. 107) of $\operatorname{str}(\sigma) \cap\left(M-M^{\prime}\right)$. (Since $M^{\prime}$ is convex, algvol is well-defined on the relative chain complex.) It follows from Stokes theorem that we get an induced map $\operatorname{algvol}_{*}: H_{n}\left(M, M^{\prime} ; \mathbb{R}\right) \rightarrow \mathbb{R}$.

Lemma 2.5. Let $M$ be a hyperbolic $n$-manifold, $M^{\prime}$ a convex subset such that algvol : $H_{n}\left(M, M^{\prime} ; \mathbb{R}\right) \rightarrow \mathbb{R}$ is an isomorphism. Let $\sum_{i \in I} a_{i} \sigma_{i} \in$ $C_{n}\left(M, M^{\prime} ; \mathbb{R}\right)$ be a straight relative $n$-cycle. Then there is a subset of indices $J \subset I$ such that all $\sigma_{j}$ with $j \in J$ are nondegenerate and $\sum_{j \in J} a_{j} \sigma_{j}$ is relatively homologous to $\sum_{i \in I} a_{i} \sigma_{i}$.

Proof. Let $K:=\left\{k \in I: \sigma_{k}\right.$ degenerate $\}$ be the set of indices of degenerate simplices occuring in $\sum_{i \in I} a_{i} \sigma_{i}$. We claim that $\sum_{k \in K} a_{k} \sigma_{k}$ is a relative 
cycle. Indeed, the degenerate faces of $\sum_{k \in K} a_{k} \sigma_{k}$ cancel each other (relatively), since they cancel in $\partial\left(\sum_{i \in I} a_{i} \sigma_{i}\right)$ and they can not cancel against faces of nondegenerate simplices. Moreover, the nondegenerate faces of degenerate simplices cancel anyway: If $\left(a, v_{1}, \ldots, v_{n}\right)$ and $\left(b, v_{1}, \ldots, v_{n}\right)$ are nondegenerate faces of a degenerate simplex, then necessarily $a=b$. Thus this face contributes twice to the boundary, with opposite signs.

We have obtained that $\sum_{k \in K} a_{k} \sigma_{k}$ is a relative cycle. But, since all $\sigma_{k}$ are degenerate, they have vanishing volume, and we have that the relative homology class $\left[\sum_{k \in K} a_{k} \sigma_{k}\right] \in \operatorname{ker}\left(\operatorname{algvol}_{*}\right)=0$ (since $\operatorname{algvol}_{*}$ is an isomorphism, by assumption), i.e., $\sum_{k \in K} a_{k} \sigma_{k} \in \operatorname{ker}($ algvol $)=0$ is a relative boundary. Then choose $J=I-K$.

In conclusion, if $M^{\prime} \subset M$ convex and $n=\operatorname{dim}(M)$, then to any relative $n$-cycle $c \in C_{n}\left(M, M^{\prime} ; \mathbb{R}\right)$ we find $c^{\prime} \in C_{n}\left(M, M^{\prime} ; \mathbb{R}\right)$ homologous to $c$ in $C_{*}\left(M, M^{\prime} ; \mathbb{R}\right)$, such that $\left\|c^{\prime}\right\| \leq\|c\|$ and $c^{\prime}$ is an alternating linear combination of nondegenerate straight simplices.

2.5.3. Straight chains as measures. We explained in 2.3 that singular chains may be considered as measures on the space of singular simplices, thus getting a homomorphism $C_{*}(M ; \mathbb{R}) \rightarrow \mathcal{C}_{*}(M)$. As we said, to get nontrivial results, we should consider not only $\mathcal{C}_{*}(M)$, but measures on the space of possibly ideal simplices. Since it is hard to prove existence of accumulation points in this measure space, we will consider measures on smaller sets of simplices.

Let $M$ be a hyperbolic manifold. The set of nondegenerate, possibly ideal, straight $i$-simplices in $M=\Gamma \backslash \mathbb{H}^{n}$ is

$$
S S_{i}(M):=\Gamma \backslash\left\{\left(p_{0}, \ldots, p_{i}\right): p_{0}, \ldots, p_{i} \in \overline{\mathbb{H}^{n}}, p_{j} \neq p_{k} \text { if } j \neq k\right\},
$$

where $g \in \Gamma$ acts by $g\left(p_{0}, \ldots, p_{n}\right)=\left(g p_{0}, \ldots, g p_{n}\right)$.

Denote $\mathcal{M}\left(S S_{i}(M)\right)$ the space of signed regular measures on $S S_{i}(M)$. Straight singular chains $c=\sum_{j=1}^{r} a_{j} \sigma_{j} \in C_{i}(M ; \mathbb{R})$, with all $\sigma_{j}$ nondegenerate, can be considered as discrete signed measures on $S S_{i}(M)$ defined by

$$
c(B)=\sum_{\left\{j: \sigma_{j} \subset B\right\}}\left|a_{j}\right|
$$

for any Borel set $B \subset S S_{i}(M)$.

Let $n=\operatorname{dim}(M)$. To apply Alaoglu's theorem to $\mathcal{M}\left(S S_{n}(M)\right)$, we need to know that $S S_{n}(M)$ is locally compact (which is obvious) and metrizable.

Lemma 2.6. Let $M$ be a hyperbolic manifold of dimension $n \geq 3$. Then $S S_{n}(M)$ is metrizable.

Proof. We have to show that $\Gamma$-orbits on $\Pi_{j=0}^{n} \overline{\mathbb{H}^{n}}-D$ are closed, $D$ being the set of degenerate straight simplices. On the complement of $\prod_{j=0}^{n} \partial_{\infty} \overline{\mathbb{H}^{n}}$ this follows from proper discontinuity of the $\Gamma$-action on $\mathbb{H}^{n}$. 
Now we assume $n \geq 3$. To any $n$-tuple $\left(v_{0}, \ldots, v_{n-1}\right) \in \Pi_{j=0}^{n-1} \partial_{\infty} \overline{\mathbb{H}^{n}}$ of distinct points corresponds a unique $v_{n} \in \partial_{\infty} \overline{\mathbb{H}^{n}}$ such that $\left(v_{0}, \ldots, v_{n}\right)$ is a positively oriented regular ideal $n$-simplex. (If $n=2$, then $v_{n}$ is not uniquely determined.) Together with 2.1 , we get a $\Gamma$-equivariant homeomorphism $\Pi_{j=0}^{n-1} \partial_{\infty} \overline{\mathbb{H}^{n}}-D \rightarrow \operatorname{Isom}^{+}\left(\mathbb{H}^{n}\right)$. Since $\Gamma \backslash \mathbb{H}^{n}$ is a manifold, we know that $\Gamma$ acts properly discontinuously on $\operatorname{Isom}^{+}\left(\mathbb{H}^{n}\right)$, thus also on $\Pi_{j=0}^{n-1} \partial_{\infty} \overline{\mathbb{H}^{n}}-D$. This implies of course that it acts properly discontinuously on $\Pi_{j=0}^{n} \partial_{\infty} \overline{\mathbb{H}^{n}}-$ $D$. Thus, $\Gamma$-orbits are closed.

\section{Degeneration.}

3.1. Efficient fundamental cycles. For a closed hyperbolic manifold $M$, we know that $\|M\|=\frac{1}{V_{n}} \operatorname{Vol}(M)$. This means that, for any $\epsilon>0$, there is some fundamental cycle $c_{\epsilon}$ satisfying $\left\|c_{\epsilon}\right\| \leq\|M\|+\frac{\epsilon}{V_{n}}$. By 2.5.1 and 2.5.2, we can choose $c_{\epsilon}$ to be an alternating chain consisting of nondegenerate straight simplices, without increasing the $l^{1}$-norm. To speak about limits of sequences of $c_{\epsilon}$, one has to regard them as elements of some locally compact space, namely the space of signed Radon measures on $S S_{n}(M)=$ $\Gamma \backslash\left(\Pi_{j=0}^{n} \overline{\mathbb{H}^{n}}-D\right)$ with the weak-*-topology, as in 2.5.3.

Jungreis' results from [11], for closed hyperbolic manifolds of dimension $\geq 3$, can be rephrased as follows:

- Any sequence of $c_{\epsilon}$ as above, with $\epsilon \rightarrow 0$, converges,

- the limit is a signed measure $\mu$, which is supported on the set of regular ideal simplices (to be identified with Isom $\left(\mathbb{H}^{n}\right)$ ), and

- up to a multiplicative factor one has $\mu=\mu^{+}-\mu^{-}$with $\mu^{+}$the Haar measure on $\operatorname{Isom}^{+}\left(\mathbb{H}^{n}\right)$ and $\mu^{-}=r^{*} \mu^{+}$for an arbitrary orientation reversing $r \in \operatorname{Isom}\left(\mathbb{H}^{n}\right)$.

The aim of this chapter is to generalize these results to finite-volume hyperbolic manifolds. For these cusped hyperbolic manifolds, there arises a technical problem: We wish to consider chains representing the relative fundamental class of a manifold with boundary, but we have a hyperbolic metric (and a notion of straightening) only on the interior. In the following, we will get around this problem and analyse the possible limits:

Let $M$ be a compact $n$-manifold with boundary $\partial M$ such that int $(M)$ carries a hyperbolic metric of finite volume. With respect to this hyperbolic metric, denote $M_{[a, b]}:=\{x \in \operatorname{int}(M): a \leq \operatorname{inj}(x) \leq b\}$. It is a wellknown consequence of the Margulis lemma ([2], D.3.12.) that, for sufficiently small $\epsilon>0$, the ' $\epsilon$-thin part' $M_{[0, \epsilon]}$ is a product neighborhood of $\partial M$, i.e., homeomorphic to $\partial M \times[0, \infty)$. Thus, one has a retraction $r_{\epsilon}$ from $M$ to the ' $\epsilon$-thick part' $M_{[\epsilon, \infty]}$ which induces a homeomorphism of pairs 
$r_{\epsilon}:(M, \partial M) \rightarrow\left(M_{[\epsilon, \infty]}, \partial M_{[\epsilon, \infty]}\right)$ and, thus, an isomorphism

$$
r_{\epsilon *}: H_{*}(M, \partial M) \rightarrow H_{*}\left(M_{[\epsilon, \infty]}, \partial M_{[\epsilon, \infty]}\right) .
$$

(This applies to all $\epsilon<\epsilon_{0}$, where $\epsilon_{0}$ depends on $M$.)

It should be noted that $M_{[0, \epsilon]}$ is convex and that one has the isomorphism

$$
\operatorname{algvol}: H_{n}\left(M, M_{[0, \epsilon]} ; \mathbb{R}\right) \rightarrow \mathbb{R} .
$$

Convexity of $M_{[0, \epsilon]}$ implies that the straightening homomorphism

$$
\text { Str : } C_{*}\left(\operatorname{int}(M), M_{[0, \epsilon]}\right) \rightarrow C_{*}\left(\operatorname{int}(M), M_{[0, \epsilon]}\right)
$$

is well-defined and induces an isomorphism in relative homology. Moreover, there is the inclusion

$$
\operatorname{exc}: C_{*}\left(M_{[\epsilon, \infty]}, \partial M_{[\epsilon, \infty]}\right) \rightarrow C_{*}\left(\operatorname{int}(M), M_{[0, \epsilon]}\right),
$$

which induces an isomorphism in homology by the excision theorem. In conclusion,

$$
\operatorname{Str}\left(\operatorname{exc}\left(r_{\epsilon *}\right)\right): C_{n}(M, \partial M ; \mathbb{R}) \rightarrow C_{n}\left(\operatorname{int}(M), M_{[0, \epsilon]} ; \mathbb{R}\right)
$$

induces an isomorphism in homology and does not increase $l^{1}$-norms.

Let, for $\epsilon<\epsilon_{0}, c_{\epsilon} \in C_{n}(M, \partial M ; \mathbb{R})$ be some relative fundamental cycle satisfying

$$
\left\|c_{\epsilon}\right\| \leq\|M, \partial M\|+\frac{\epsilon}{V_{n}} .
$$

By the above arguments, we may replace $c_{\epsilon}$ by

$$
\operatorname{Str}\left(\operatorname{exc}\left(r_{\epsilon *} c_{\epsilon}\right)\right) \in C_{n}\left(\operatorname{int}(M), M_{[0, \epsilon]} ; \mathbb{R}\right)
$$

without increasing the $l^{1}$-norm. Abusing notation, we will continue to denote this new relative cycle by $c_{\epsilon}$.

Definition 3.1. A signed measure $\mu$ on $S S_{n}(M)$ is called an efficient fundamental chain if there exists a sequence of $\epsilon$ with $\epsilon \rightarrow 0$ and a sequence of $c_{\epsilon} \in C_{n}(M, \partial M ; \mathbb{R})$ representing the relative fundamental class $[M, \partial M]$, which are alternating chains invoking only nondegenerate simplices and which satisfy $\left\|c_{\epsilon}\right\| \leq\|M, \partial M\|+\frac{\epsilon}{V_{n}}$, such that the sequence $\operatorname{Str}\left(\operatorname{exc}\left(r_{\epsilon *} c_{\epsilon}\right)\right)$ $\in C_{n}\left(\operatorname{int}(\operatorname{int}(M)), M_{[0, \epsilon]} ; \mathbb{R}\right)$ converges to $\mu$ in the weak-*-topology of $\mathcal{M}\left(S S_{n}(M)\right)$, the space of signed measures on the space of straight nondegenerate simplices.

Lemma 3.2. Assume that $M$ is a manifold of dimension $n \geq 3$, such that $\operatorname{int}(M)$ admits a hyperbolic metric of finite volume. Then there is at least one efficient fundamental chain. 
Proof. Considering some sequence of $c_{\epsilon}$ with $\epsilon \rightarrow 0$, we may by Lemma 2.5 assume that the support of the $c_{\epsilon}$ consists of only straight nondegenerate simplices. $\operatorname{Str}\left(\operatorname{exc}\left(r_{\epsilon *} c_{\epsilon}\right)\right)$ may be regarded as a sequence of signed measures on the locally compact metric space $S S_{n}(M)$, see 2.5.3. The sequence $c_{\epsilon}$ is bounded by its definition and, hence, Lemmas 2.2 and 2.6 guarantee the existence of a weak-*-accumulation point $\mu$. (The condition $n \geq 3$ is needed to apply Lemma 2.6.)

We recall that excision and straightening, as well as the homeomorphism $r_{\epsilon}$ induce isomorphisms in relative homology. Hence, any new $c_{\epsilon}$ represents the relative fundamental class in $H_{n}\left(\operatorname{int}(M), M_{[0, \epsilon]} ; \mathbb{R}\right)$. As a special case of Lemma 2.4 we have:

Lemma 3.3. Let $c_{\epsilon}$ be a representative of the relative fundamental class $\left[\operatorname{int}(M), M_{[0, \epsilon]}\right]$. Then $\Phi_{x}\left(c_{\epsilon}\right)=1$ holds for almost all $x \in M_{[\epsilon, \infty]}$.

Definition 3.4. For a hyperbolic manifold $M$, let $S_{\delta} \subset S S_{n}(M)$ be the set of nondegenerate straight simplices $\sigma \in M$ with $\operatorname{vol}(\sigma)<V_{n}-\delta$.

Lemma 3.5. An efficient fundamental chain $\mu$ is supported on $S S_{n}(M)-$ $S_{0}$, i.e., on the set of straight simplices of volume $V_{n}$.

Proof. It suffices to show that $\mu\left(S_{\delta}\right)=0$ holds for any $\delta>0$. By Lemma 2.2, (ii), and openness of $S_{\delta}$, this follows if we can prove $\lim _{\epsilon \rightarrow 0} c_{\epsilon}\left(S_{\delta}\right)=0$ for any $\delta>0$. Here, $c_{\epsilon}=\sum_{j=1}^{r} a_{j} \sigma_{j}$ is the sequence from Definition 3.1.

From Lemma 3.3, we conclude $\int_{M} \Phi_{x}\left(c_{\epsilon}\right) d \operatorname{vol}(x) \geq \operatorname{Vol}\left(M_{[\epsilon, \infty]}\right)$. But $\int_{M} \Phi_{x}\left(c_{\epsilon}\right) d \operatorname{vol}(x)=\sum_{j=1}^{r} a_{j} \int_{M} \Phi_{x}\left(\sigma_{j}\right) d \operatorname{vol}(x)=\sum_{j=1}^{r} a_{j}$ algvol $\left(\sigma_{j}\right)$, where $\operatorname{algvol}\left(\sigma_{j}\right)$ is $\operatorname{Vol}\left(\sigma_{j}\right)$ with a sign according to orientation. As a consequence:

$$
\sum\left|a_{j}\right| \operatorname{Vol}\left(\sigma_{j}\right) \geq \operatorname{Vol}\left(M_{[\epsilon, \infty]}\right) .
$$

On the other hand, we want $c_{\epsilon}=\sum_{j=1}^{r} a_{j} \sigma_{j}$ to satisfy $V_{n} \sum\left|a_{j}\right| \leq$ $\mathrm{Vol}(M)+\epsilon$. Subtracting the two inequalities yields

$$
\sum\left|a_{j}\right|\left(V_{n}-\operatorname{Vol}\left(\sigma_{j}\right)\right) \leq \epsilon+\operatorname{Vol}\left(M_{[0, \epsilon]}\right) .
$$

We get

$$
\begin{aligned}
\epsilon & +\operatorname{Vol}\left(M_{[0, \epsilon]}\right) \\
\geq & \sum\left|a_{j}\right|\left(V_{n}-\operatorname{Vol}\left(\sigma_{j}\right)\right) \\
= & \sum_{j: \operatorname{Vol}\left(\sigma_{j}\right) \geq V_{n}-\delta}\left|a_{j}\right|\left(V_{n}-\operatorname{Vol}\left(\sigma_{j}\right)\right) \\
& +\sum_{j: \operatorname{Vol}\left(\sigma_{j}\right)<V_{n}-\delta}\left|a_{j}\right|\left(V_{n}-\operatorname{Vol}\left(\sigma_{j}\right)\right)
\end{aligned}
$$




$$
\begin{aligned}
& \geq \sum_{j: \operatorname{Vol}\left(\sigma_{j}\right)<V_{n}-\delta}\left|a_{j}\right|\left(V_{n}-\operatorname{Vol}\left(\sigma_{j}\right)\right) \\
& \geq \delta \sum_{j: \operatorname{Vol}\left(\sigma_{j}\right)<V_{n}-\delta}\left|a_{j}\right| \\
& =\delta c_{\epsilon}\left(S_{\delta}\right) .
\end{aligned}
$$

Since $\lim _{\epsilon \rightarrow 0} \operatorname{Vol}\left(M_{[0, \epsilon]}\right)=0$, we conclude $\lim _{\epsilon \rightarrow 0} c_{\epsilon}\left(S_{\delta}\right)=0$.

We have just proved that, if $c_{\epsilon}$ is a representative of $\left[\operatorname{int}(M), M_{[0, \epsilon]}\right]$ satisfying $V_{n}\left\|c_{\epsilon}\right\| \leq \operatorname{Vol}(M)+\epsilon$, then $\lim _{\epsilon \rightarrow 0} c_{\epsilon}\left(S_{\delta}\right)=0$ holds for any $\delta>0$. Since $\left\|c_{\epsilon}^{ \pm}\right\| \leq\left\|c_{\epsilon}\right\|$ and $\int_{M} \Phi_{x}\left(c_{\epsilon}^{ \pm}\right) \geq \operatorname{Vol}\left(M_{[\epsilon, \infty]}\right)$, we can use the same argument to prove that $\lim _{\epsilon \rightarrow 0} c_{\epsilon}^{ \pm}\left(S_{\delta}\right)=0$ holds for any $\delta>0$. This fact will be used in the proof of the following lemma:

Lemma 3.6. Let $\mu$ be an efficient fundamental chain. Then $\mu \neq 0$.

Proof. Choose a continuous $f: S S_{n}(M) \rightarrow[0,1]$, which vanishes on some $S_{\delta^{\prime}}$ and is 1 on the complement of some $S_{\delta}$. As $f$ is compactly supported, we have $\mu(f)=\lim _{\epsilon \rightarrow 0} c_{\epsilon}(f)$. (Here we use that we are admitting ideal simplices: Otherwise the support of $f$ would not be compact.)

Now using $\lim _{\epsilon \rightarrow 0} c_{\epsilon}\left(S_{\delta}\right)=0$ we have

$$
\mu^{ \pm}(f)=\lim _{\epsilon \rightarrow 0} c_{\epsilon}^{ \pm}(f) \geq \lim _{\epsilon \rightarrow 0} c_{\epsilon}^{ \pm}\left(S S_{n}(M)\right)-c_{\epsilon}^{ \pm}\left(S_{\delta}\right)=\lim _{\epsilon \rightarrow 0} c_{\epsilon}^{ \pm}\left(S S_{n}(M)\right) .
$$

But $c_{\epsilon}^{+}\left(S S_{n}(M)\right)+c_{\epsilon}^{-}\left(S S_{n}(M)\right)=\left\|c_{\epsilon}^{+}\right\|+\left\|c_{\epsilon}^{-}\right\|=\left\|c_{\epsilon}\right\| \geq\|M, \partial M\|$ implies that one of $\lim _{\epsilon \rightarrow 0} c_{\epsilon}^{ \pm}\left(S S_{n}(M)\right)$ must be at least $\frac{1}{2}\|M, \partial M\|$, thus positive.

Lemma 3.7. Efficient fundamental chains $\mu$ are cycles, i.e., $(\partial \mu)^{+}=(\partial \mu)^{-}$ $=0$.

Proof. Denote by $T_{\epsilon}^{i}(M)$ the set of (possibly ideal) $i$-simplices intersecting $M_{[\epsilon, \infty]}$. For all $\delta<\epsilon$, we get by the convexity of $M_{[0, \delta]} \subset M_{[0, \epsilon]}$ :

$$
B \subset T_{\epsilon}^{n-1}(M) \text { measurable } \Rightarrow \partial c_{\delta}^{ \pm}(B)=0 .
$$

When $\partial \mu^{ \pm}$is a weak-* accumulation point of a sequence $\partial c_{\delta}^{ \pm}$, we conclude $\partial \mu^{ \pm}(B)=0$ for all measurable sets $B$ contained in some $T_{\epsilon}^{n-1}(M)$ by Part (ii) of Lemma 2.2, since we may consider them as subsets of an open set still contained in some slightly larger $T_{\epsilon}^{n-1}(M)$.

But clearly, $\cup_{k=1}^{\infty} T_{\frac{1}{k}}^{n-1}(M)$ is the set of all (even ideal) $(n-1)$-simplices, hence the claim of the lemma.

Remark. In the case of closed manifolds, this lemma is, of course, an immediate consequence of the fact that $\|\partial\| \leq n+1$. 


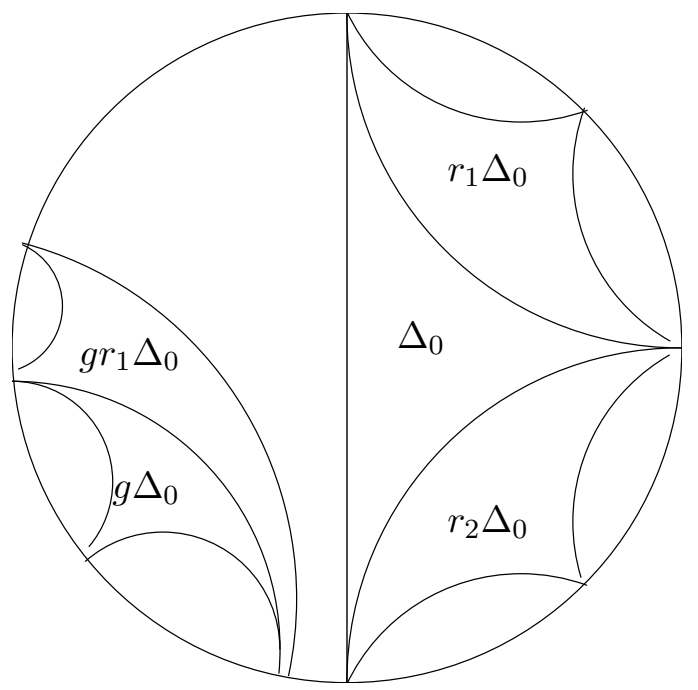

3.2. Invariance under ideal reflection group. Since we have an ordering of the vertices of a simplex $\Delta$, we can speak of the $i$-th face of $\Delta$, the codimension 1-face not containing the $i$-th vertex.

Definition 3.8. Fix a regular ideal simplex $\Delta_{0} \subset \mathbb{H}^{n}$ and, for $i=0, \ldots, n$, let $r_{i}$ be the reflection in the $i$-th face of $\Delta_{0}$. Let $R \subset \operatorname{Isom}\left(\mathbb{H}^{n}\right)$ be the subgroup generated by $r_{0}, \ldots, r_{n}$ and let $R^{+}=R \cap \operatorname{Isom}^{+}\left(\mathbb{H}^{n}\right)$.

We know that $\mu^{ \pm}$are measure cycles supported on the set of regular ideal simplices. By 2.1 , we may consider $\mu^{ \pm}$as measures on $\Gamma \backslash \operatorname{Isom}\left(\mathbb{H}^{n}\right)$, after fixing some regular ideal simplex $\Delta_{0}$ in $\mathbb{H}^{n}$.

We will use the convention that $\gamma \in \operatorname{Isom}\left(\mathbb{H}^{n}\right)$ corresponds to the simplex $\gamma \Delta_{0}$, i.e., we let $\operatorname{Isom}\left(\mathbb{H}^{n}\right)$, and in particular $\Gamma$, act from the left. It will be important to note that, after this identification, the right-hand action of $R$ corresponds to the following operation on the set of simplices: $r_{i}$ maps a simplex to the simplex obtained by reflection in the $i$-th face. This is clear from the picture above.

Lemma 3.9. For $n \geq 3$, efficient fundamental chains are invariant under the right-hand action of $R^{+}$on $\Gamma \backslash \operatorname{Isom}\left(\mathbb{H}^{n}\right)$.

Note. If $\Delta=g \Delta_{0}$ for some $g \in \Gamma \backslash \operatorname{Isom}\left(\mathbb{H}^{n}\right)$, then the reflection $s_{i}$ in the $i$-th face of $\Delta$ maps $\Delta=g \Delta_{0}$ to $g r_{i}\left(\Delta_{0}\right)$. In other words, the choice of another reference simplex changes the identification with Isom $\left(\mathbb{H}^{n}\right)$ by left multiplication with $g \in \operatorname{Isom}\left(\mathbb{H}^{n}\right)$, but does not alter the right-hand action of $R^{+}$on Isom $\left(\mathbb{H}^{n}\right)$. This implies that the truth of Lemma 3.9 is independent of the choice of $\Delta_{0}$.

Lemma 3.9 follows from:

Lemma 3.10. In dimensions $n \geq 3$, a signed alternating measure $\mu$ on the set of maximal volume simplices is a cycle iff $r_{i}^{*}(\mu)=-\mu$ for all $i=0, \ldots, n$. 
Proof. If $n \geq 3$, then for any ordered regular ideal $(n-1)$-simplex $\tau$, there are exactly two ordered regular ideal $n$-simplices, $\tau_{i}^{+}$and $\tau_{i}^{-}$, having $\tau$ as $i$-th face. (By the way, this is besides Lemma 2.6 and its 'corollary' Lemma 3.2 the only point entering the proofs of our theorems which uses $n \geq 3$.) We fix them such that $\tau_{i}^{+}$is positively oriented. For a measurable set $B \subset$ \{ordered regular ideal $(n-1)$-simplices\} define

$$
B_{i}^{+}=\left\{\tau_{i}^{+}: \tau \in B\right\} \text { and } B_{i}^{-}=\left\{\tau_{i}^{-}: \tau \in B\right\} .
$$

Since $\mu$ is supported on the set of regular ideal $n$-simplices, we have that

$$
\begin{aligned}
\partial \mu^{ \pm}(B) & =\sum_{k=0}^{n}(-1)^{k} \mu^{ \pm}\left(\partial_{k}^{-1}(B)\right) \\
& =\sum_{k=0}^{n}(-1)^{k}\left(\mu^{ \pm}\left(B_{k}^{+}\right)+\mu^{ \pm}\left(B_{k}^{-}\right)\right) .
\end{aligned}
$$

We may assume that $\mu$ is alternating, in particular $\pi_{i k}^{*} \mu=(-1)^{i-k} \mu$, where $\pi_{i k}$ is induced by the affine map realizing the transposition of the $i$-th and $k$-th vertex. $\pi_{i k}$ maps $B_{i}^{+}$to $B_{k}^{+}$and $B_{i}^{-}$to $B_{k}^{-}$. Therefore, for any $i \in$ $\{0, \ldots, n\}$, we get

$$
\begin{aligned}
\partial \mu^{ \pm}(B) & =\sum_{k=0}^{n}(-1)^{k}(-1)^{i-k}\left(\pi_{i k}^{*} \mu^{ \pm}\left(B_{k}^{+}\right)+\pi_{i k}^{*} \mu^{ \pm}\left(B_{k}^{-}\right)\right) \\
& =\sum_{k=0}^{n}(-1)^{i}\left(\mu^{ \pm}\left(B_{i}^{+}\right)+\mu\left(B_{i}^{-}\right)\right) \\
& =(-1)^{i}(n+1)\left(\mu^{ \pm}\left(B_{i}^{+}\right)+\mu^{ \pm}\left(B_{i}^{-}\right)\right) .
\end{aligned}
$$

In particular $\partial \mu(B)=0$ holds if and only if $\mu\left(B_{i}^{+}\right)=-\mu\left(B_{i}^{-}\right)$for $i=$ $0, \ldots, n$.

The action of $r_{i}$ maps $B_{i}^{+}$bijectively to $B_{i}^{-}$and vice versa. This implies the 'if'-part of Lemma 3.10.

To get the 'only if'-part, we use that $\partial \mu=0$ implies that $r_{i}^{*} \mu\left(B_{i}^{ \pm}\right)=$ $-\mu\left(B_{i}^{ \pm}\right)$holds, for any set $B \subset$ \{ordered regular ideal $(n-1)$-simplices $\}$. Now let $C \subset$ \{ordered regular ideal $n$-simplices $\}$ be an arbitrary set. We divide $C=C^{+} \cup C^{-}$, where $C^{+}=\{\sigma \in C: \sigma$ positively oriented $\}$. Consider $B:=\left\{\partial_{i} \sigma: \sigma \in C^{+}\right\}$. ( $i$ is arbitrary, e.g., $i=0$.) Then we have $B_{i}^{+}=C^{+}$, because for any ordered regular ideal $(n-1)$-simplex $\partial_{i} \sigma \in B, \sigma$ is the unique positively oriented ordered regular ideal $n$-simplex having $\partial_{i} \sigma$ as its $i$-th face. Thus $\mu\left(C^{+}\right)=-r_{i}^{*}\left(C^{+}\right)$. The same way one gets $\mu\left(C^{-}\right)=-r_{i}^{*} \mu\left(C^{-}\right)$, thus $\mu(C)=-r_{i}^{*} \mu(C)$. Since $C$ was arbitrary, this proves the 'only if'-part.

Remark. A different proof of the same fact is given in Lemma 2.2 of [11]. 


\section{Decomposition of efficient fundamental cycles.}

If $n \geq 4$, then the group generated by reflections in the faces of a regular ideal $n$-simplex in $\mathbb{H}^{n}$ is dense in Isom $\left(\mathbb{H}^{n}\right)$. We get therefore from Lemma 3.9 that efficient fundamental cycles are invariant under the right-hand action of $\operatorname{Isom}^{+}\left(\mathbb{H}^{n}\right)$. This implies that they are a multiple of Haar $-r^{*}$ Haar, where Haar is the Haar measure on $\operatorname{Isom}^{+}\left(\mathbb{H}^{n}\right)$. (It is well-known that all invariant measures on a Lie group are multiples of the Haar measure.)

In the following we will discuss the case $n=3$.

We wish to recall some facts from the ergodic theory of unipotent actions.

The Iwasawa decomposition $G=K A N$ of $G=\mathrm{Isom}^{+}\left(\mathbb{H}^{n}\right)$ is as follows: Fix some $v_{\infty} \in \partial_{\infty} \mathbb{H}^{n}$ and some $p \in \mathbb{H}^{n}$. Then we may take $K$ to be the group of isometries fixing $p, A$ the group of translations along the geodesic through $p$ and $v_{\infty}$, and $N$ the group of translations along the horosphere through $p$ and $v_{\infty}$.

We will consider the natural right-hand action of $N$ on $G=K A N$.

The next lemma follows from [5]. It is nowadays a special case of the Ragunathan conjecture, which was proved by Ratner.

Lemma 4.1. Let $G=K A N$ be the Iwasawa decomposition of a simple Lie group of $\mathbb{R}$-rank 1 , and $\Gamma \subset G$ a discrete subgroup of finite covolume. If $\mu$ is a finite $N$-invariant ergodic measure on $\Gamma \backslash G$, then $\mu$ is either a multiple of the Haar measure or it is supported on a compact $N$-orbit.

The following lemma is a straightforward generalisation of Theorem 4.4. in $[6]$ :

Lemma 4.2. Let $G=K A N$ be the Iwasawa decomposition of a simple Lie group of $\mathbb{R}$-rank 1 , and $\Gamma \subset G$ a discrete subgroup of finite covolume. Let $N^{\prime} \subset N$ be a closed subgroup such that $N / N^{\prime}$ is compact. Then any $N^{\prime}$ invariant ergodic measure on $\Gamma \backslash G$ is either a multiple of the Haar measure or is supported on a compact $N$-orbit.

Proof. We will use several times the following basic fact: If $G_{1}$ and $G_{2}$ are subgroups of a group $G$ endowed with a measure $\mu$, then the left action of $G_{1}$ on $G / G_{2}$ is ergodic if and only if the right action of $G_{2}$ on $G_{1} \backslash G$ is ergodic. (This is known as Moore-equivalence.)

By Moore-equivalence, ergodic measures for the $N^{\prime}$-action on $\Gamma \backslash G$ correspond to ergodic measures for the action of $\Gamma$ on $G / N^{\prime}$. Consider, therefore, $\mu$ as a measure on $G / N^{\prime}$, ergodic with respect to the $\Gamma$-action. Let pr : $G / N^{\prime} \rightarrow G / N$ be the projection. Since $N / N^{\prime}$ is compact, we have a locally finite measure $\operatorname{pr}_{*} \mu$ on $G / N$ which is easily seen to be ergodic with respect to the $\Gamma$-action. By Lemma 4.1 and Moore-equivalence, $\mathrm{pr}_{*} \mu$ must either be the Haar measure or correspond to an $N$-invariant measure on $\Gamma \backslash G$ which is determined on a compact orbit $\Gamma \backslash \Gamma g N \subset \Gamma \backslash G$. 
If $\operatorname{pr}_{*} \mu=$ Haar measure, it follows easily that $\mu$ is absolutely continuous with respect to the Haar measure and then one gets, from ergodicity of the $\Gamma$-action (Theorem 7 in [14]), that $\mu$ is a multiple of the Haar measure.

In the second case, $\operatorname{pr}_{*} \mu$ must be supported on the $\Gamma$-orbit of some $g N \in$ $G / N$. Therefore, $\mu$ is supported on the $\Gamma \times N$-orbit of $g N^{\prime} \in G / N^{\prime}$. By Moore-equivalence we get a measure supported on the compact $N$-orbit.

Lemmas 4.1 and 4.2 apply in particular to $G=\operatorname{Isom}^{+}\left(\mathbb{H}^{n}\right)$ with the Iwasawa decomposition described above.

Back to the situation of Section 3.2. Let $v$ be an ideal vertex of the reference simplex $\Delta_{0}$. Let $N_{v} \subset \operatorname{Isom}^{+}\left(\mathbb{H}^{3}\right)$ be the subgroup of parabolic isometries fixing $v$. We may consider $N_{v}$ as the $N$-factor in the Iwasawa decomposition Isom ${ }^{+}\left(\mathbb{H}^{3}\right)=K_{v} A_{v} N_{v}$. (That means we use $v$ and some arbitrary $p \in \mathbb{H}^{3}$ to construct the Iwasawa decomposition. In the following, we will fix some arbitrary $p \in \mathbb{H}^{3}$ but consider various $v \in \partial_{\infty} \mathbb{H}^{3}$, therefore the labelling of the Iwasawa decompositions.)

Instead of $R^{+}$defined in Section 3.2, we consider only the subgroup $T_{v}^{\prime} \subset$ $R^{+} \subset$ Isom $^{+}\left(\mathbb{H}^{3}\right)$ generated by products of even numbers of reflections in those faces of $\Delta_{0}$ which contain $v . \mu$ is, of course, also invariant under the smaller group $T_{v}^{\prime}$. In [11] it is shown that $T_{v}^{\prime}$ contains a subgroup $T_{v}$ which is a cocompact subgroup of $N_{v}$ (if $n=3$ ).

The signed measure $\mu$ decomposes as a difference of two (nonnegative) measures $\mu^{+}$and $\mu^{-}$. Both are invariant under the right-hand action of $T_{v}$. From Lemma 2.1 , we get that the probability measures $\bar{\mu}^{ \pm}$, obtained by rescaling the restrictions of $\mu^{ \pm}$to $\Gamma \backslash \operatorname{Isom}^{+}\left(\mathbb{H}^{3}\right)$, have decomposition maps with respect to the action of $T_{v}$,

$$
\beta_{v}^{ \pm}: \Gamma \backslash \operatorname{Isom}^{+}\left(\mathbb{H}^{3}\right) \rightarrow \mathcal{E} .
$$

Here, $\mathcal{E}$ is the set of ergodic $T_{v}$-invariant measures on $\Gamma \backslash \operatorname{Isom}^{+}\left(\mathbb{H}^{3}\right)$. From Lemma 4.2, we get that $\mathcal{E}$ consists of Haar (the Haar measure, rescaled to a probability measure) and measures determined on compact $N_{v}$-orbits. The following lemma is well-known:

Lemma 4.3. An orbit $g N_{v}$ is compact in $\Gamma \backslash \operatorname{Isom}\left(\mathbb{H}^{n}\right)$ iff all simplices $g h \Delta_{0}$ with $h \in N_{v}$ have its ideal vertex $g(v)$ in a parabolic fixed point of $\Gamma$.

Proof. Parametrise elements of $N_{v}$ as $u(s), s \in \mathbb{R}^{n-1}$ (identifying a stabilized horosphere with euclidean $(n-1)$-space). The $N_{v}$-orbit of $g$ on $\Gamma \backslash \operatorname{Isom}\left(\mathbb{H}^{n}\right)$ is compact if and only if, for all $s \in \mathbb{R}^{n-1}$, one finds $\gamma \in \Gamma$ and $t \in \mathbb{R}$ such that $g u(t s)=\gamma g$. This $\gamma$ is then conjugated to $u(t s)$ and, in particular, is parabolic, i.e., has only one fixed point. The fixed point of $\gamma$ must be $g(v)$, since $\gamma g(v)=g u(t s)(v)=g(v)$.

The other implication is straightforward. 
To summarize, we have the following statement: For any vertex $v$ of the reference simplex $\Delta_{0}$, the ergodic decomposition of the rescaled $\bar{\mu}^{ \pm}$with respect to the right-hand action of $T_{v}$ uses the Haar measure and measures determined on the set of those simplices $g \Delta_{0}$ which have the vertex $g(v)$ in a parabolic fixed point of $\Gamma$.

\subsection{Manifolds which are not Gieseking-like.}

Definition 4.4. A 3-manifold is Gieseking-like if it has a hyperbolic structure $M=\Gamma \backslash \mathbb{H}^{3}$ of finite volume such that $Q(\omega) \cup\{\infty\} \subset \partial_{\infty} \mathbb{H}^{3}$ are parabolic fixed points of $\Gamma$.

Here, we have used the upper half space model of $\mathbb{H}^{3}$, and identified the ideal boundary with $\mathbb{C} \cup\{\infty\} . \omega=\frac{1}{2}+\frac{\sqrt{-3}}{2}$ is the 4 th vertex of a regular ideal simplex with vertices $0,1, \infty$. The condition is, of course, equivalent to the condition that $\Gamma$ is conjugate to a discrete subgroup of $\operatorname{PSL}_{2} Q(\omega)$ after the identification of $\operatorname{Isom}^{+}\left(\mathbb{H}^{3}\right)$ with $\mathrm{PSL}_{2} \mathbb{C}$. One does not seem to know any example of a Gieseking-like manifold which is not a finite cover of the Gieseking manifold (communicated to the author by Alan Reid, see also $[\mathbf{1 3}])$.

Theorem 4.5. Let $M$ be a compact manifold of dimension $n \geq 3$ such that $\operatorname{int}(M)$ admits a hyperbolic metric of finite volume. Assume that $M$ is either of dimension $\geq 4$ or that $M$ is of dimension 3 and is not Gieseking-like.

If $\mu$ is an efficient fundamental cycle on $M$, then $\mu=K$ (Haar $-r^{*}$ Haar) for some real number $K$.

Proof. By the first remark of Chapter 4, we may restrict to dimension 3. We have to exclude the existence of a signed measure $\nu$ which is supported on the set of regular ideal simplices with vertices in cusps and which satisfies $r^{*} \nu= \pm \nu$ for all $r \in R$. However, the existence of such a nontrivial signed measure would imply the existence of an $R$-invariant family $\{\Delta r: r \in R\}$ of simplices with vertices in the cusps of $M=\Gamma \backslash \mathbb{H}^{3}$. By 2.1, there is $g \in \operatorname{Isom}\left(\mathbb{H}^{3}\right)$ with $\Delta=g \Delta_{0}$, where $\Delta_{0}$ is the ideal simplex with vertices $0,1, \infty, \omega$ in the upper half-space model. We get that all vertices of the form $g v_{\infty} r$ with $r \in R$ and $v_{\infty}$ one of $0,1, \infty, \omega$ must be parabolic fixed points of $\Gamma$. Note that $\left\{v_{\infty} r: v_{\infty} \in\{0,1, \omega, \infty\}, r \in R\right\}=Q(\omega) \cup\{\infty\}$. Thus, conjugating $\Gamma$ with $g$ we get a hyperbolic structure with all of $Q(\omega) \cup\{\infty\}$ as parabolic fixed points.

\section{Cycles not transversal to geodesic surfaces.}

In the last section, we classified efficient fundamental cycles on finite-volume hyperbolic manifolds which are not Gieseking-like. In this chapter, we will see that for arbitrary (possibly Gieseking-like) finite-volume hyperbolic manifolds we can still obtain information which in Chapter 6 will be used to derive glueing inequalities. 
Definition 5.1. For a hyperbolic manifold $M$ and a two-sided totally geodesic codimension-1 submanifold $F \subset M$ call:

- $S_{\text {cusp }}^{i}$ the set of positively oriented ideal $i$-simplices with all vertices in parabolic fixed points of $M$, and

- $S_{F}^{i}$ the set of (possibly ideal) positively oriented $i$-simplices that intersect $\mathrm{F}$ transversally.

Here, a simplex $\sigma$ is said to intersect $F$ transversally if it intersects both components of any regular neighborhood of $F$.

Lemma 5.2. If $M$ is a hyperbolic manifold and $F$ is a two-sided totally geodesic codimension-1-submanifold, then

$$
S_{F}^{n} \cap\{\text { regular ideal simplices }\} \subset\{\text { regular ideal simplices }\}
$$

has positive Haar measure.

Proof. It is easy to see that $S_{F}^{n} \cap$ \{regular ideal simplices\} is an open, nonempty subset of \{regular ideal simplices\}.

Theorem 5.3. Let $M$ be a compact manifold of dimension $n \geq 3$ such that $\operatorname{int}(M)$ admits a hyperbolic metric of finite volume, and let $F \subset M$ be a closed totally geodesic codimension-1-submanifold.

If $\mu$ is an efficient fundamental cycle (with $\left.\left.\mu^{+}\right|_{\Gamma \backslash \operatorname{Isom}^{+}\left(\mathbb{H}^{n}\right)} \neq 0\right)$, then $\mu^{+}\left(S_{F}^{n}\right) \neq 0$.

Proof. Very roughly, the idea is the following: If $\mu^{+}\left(S_{F}^{n}\right)$ vanishes, then the Haar measure can only give a zero contribution to the ergodic decomposition of $\mu^{+}$, hence, $\mu^{+}$is supported on $S_{\text {cusp }}^{n}$. In particular, $\mu^{+}$vanishes on the set of simplices with boundary faces in $F$, and this will give a contradiction.

Rescale $\left.\mu^{+}\right|_{\Gamma \backslash \operatorname{Isom}^{+}\left(\mathbb{H}^{n}\right)}$ to a probability measure $\bar{\mu}^{+}$. 0 .

Assume for some totally geodesic surface $F$ we had $\bar{\mu}^{+}\left(S_{F}^{n}\right)=\mu^{+}\left(S_{F}^{n}\right)=$

Let $v$ be a vertex of the reference simplex $\Delta_{0}$. Using the ergodic decomposition with respect to the $T_{v}$-action on $\Gamma \backslash G=\Gamma \backslash \operatorname{Isom}^{+}\left(\mathbb{H}^{n}\right)$ yields

$$
\begin{aligned}
0=\bar{\mu}^{+}\left(S_{F}^{n}\right) & =\int_{\Gamma \backslash G} \beta_{v}(g)\left(S_{F}^{n}\right) d \bar{\mu}^{+}(g) \\
& \geq \int_{g \in \Gamma \backslash G: \beta_{v}(g)=\text { Haar }} \beta_{v}(g)\left(S_{F}^{n}\right) d \bar{\mu}^{+}(g) \\
& =\int_{g \in \Gamma \backslash G: \beta_{v}(g)=\text { Haar }} \operatorname{Haar}\left(S_{F}^{n}\right) d \bar{\mu}^{+}(g) \\
& =\operatorname{Haar}\left(S_{F}^{n}\right) \int_{g \in \Gamma \backslash G: \beta_{v}(g)=\text { Haar }} d \bar{\mu}^{+}(g) .
\end{aligned}
$$


By Lemma 5.2, Haar $\left(S_{F}^{n}\right) \neq 0$ and, thus,

$$
\int_{g \in \Gamma \backslash G: \beta_{v}(g)=\text { Haar }} d \bar{\mu}^{+}(g)=0 .
$$

We will conclude that $\mu^{+}$is supported on $S_{\text {cusp }}^{n}$ by means of Lemma 5.5, which we state separately because it will be of independent use in Chapter 7 .

Definition 5.4. Let $\Gamma \subset G=\mathrm{Isom}^{+}\left(\mathbb{H}^{n}\right)$ be a cocompact discrete subgroup, $v \in \partial_{\infty} \mathbb{H}^{n}, T_{v} \subset \operatorname{Isom}^{+}\left(\mathbb{H}^{n}\right)$ the subgroup defined in Chapter 4 and $\beta$ a decomposition map for the right-hand action of $T_{v}$, as defined in Chapter 4. Define

$$
H_{v}=\left\{g \in \Gamma \backslash G: \beta_{v}(g)=\text { Haar }\right\} .
$$

Lemma 5.5. Let $v_{0}, \ldots, v_{n}$ be the vertices of a regular ideal simplex in $\mathbb{H}^{n}$ and $\bar{\mu}^{+}$a probability measure on $\Gamma \backslash G:=\Gamma \backslash \operatorname{Isom}^{+}\left(\mathbb{H}^{n}\right)$, invariant with respect to the right-hand action of $R^{+}$. If $\bar{\mu}^{+}\left(H_{v_{i}}\right)=0$ for $i=0, \ldots, n$, then $\bar{\mu}^{+}$is supported on $S_{\text {cusp }}^{n}$.

Proof. Let

$$
A_{i}=\left\{g \in \Gamma \backslash G: g v_{i} \text { is cusp of } \Gamma\right\}
$$

and

$$
B_{i}=\left\{g \in \Gamma \backslash G: \Gamma \backslash \Gamma g N_{v_{i}} \text { is compact }\right\} .
$$

We have

$$
\Gamma \backslash G-S_{\text {cusp }}^{n}=\Gamma \backslash G-\cup_{j=0}^{n} A_{j}=\Gamma \backslash G-\cup_{j=0}^{n} B_{j}=\cap_{j=0}^{n} \Gamma \backslash G-B_{j},
$$

where the second equality holds by Lemma 4.3.

If $e$ is a $T_{v_{i}}$-ergodic measure supported on a compact $N_{v_{i}}$-orbit, then

$$
e\left(\Gamma \backslash G-B_{i}\right)=0 .
$$

Thus (abbreviating $\beta_{g}:=\beta_{v_{i}}(g)$ ),

$$
\begin{aligned}
& \bar{\mu}^{+}\left(\Gamma \backslash G-B_{i}\right) \\
& =\int_{\Gamma \backslash G} \beta_{g}\left(\Gamma \backslash G-B_{i}\right) d \bar{\mu}^{+}(g) \\
& =\int_{H_{v_{i}}} \beta_{g}\left(\Gamma \backslash G-B_{i}\right) d \bar{\mu}^{+}(g)+\int_{\Gamma \backslash G-H_{v_{i}}} \beta_{g}\left(\Gamma \backslash G-B_{i}\right) d \bar{\mu}^{+}(g) \\
& =\operatorname{Haar}\left(\Gamma \backslash G-B_{i}\right) \bar{\mu}^{+}\left(H_{v_{i}}\right)+\int_{\Gamma \backslash G-H_{v_{i}}} \beta_{g}\left(\Gamma \backslash G-B_{i}\right) d \bar{\mu}^{+}(g) \\
& =\operatorname{Haar}\left(\Gamma \backslash G-B_{i}\right) \mathbf{0}+\int_{\Gamma \backslash G-H_{v_{i}}} \mathbf{0} d \bar{\mu}^{+}(g) \\
& =\mathbf{0}
\end{aligned}
$$


and, therefore,

$$
\begin{aligned}
\bar{\mu}^{+}\left(\Gamma \backslash G-S_{\text {cusp }}^{n}\right) & =\bar{\mu}^{+}\left(\cup_{i=0}^{n} \Gamma \backslash G-B_{i}\right) \\
& \leq \sum_{i=0}^{n} \bar{\mu}^{+}\left(\Gamma \backslash G-B_{i}\right)=0 .
\end{aligned}
$$

We are now going to finish the proof of Theorem 5.3:

We know (from the proof of Lemma 3.3) that $\Phi_{x}\left(c_{\epsilon}^{+}\right) \geq \Phi_{x}\left(c_{\epsilon}\right) \geq 1$ for all $x \in M_{[\epsilon, \infty]}$. F is a closed totally geodesic hypersurface. Therefore $F \subset M_{[\epsilon, \infty]}$ for sufficiently small $\epsilon$. We conclude $\Phi_{x}\left(c_{\epsilon}^{+}\right) \geq 1$ for all $x \in F$.

For $x \in M$ let $S_{x}^{n}$ be the set of straight $n$-simplices $\Delta$ containing $x$ in their image. If $x \in F$ is contained in the totally geodesic submanifold $F$, then $S_{x}^{n}$ is the union of the following two sets of simplices:

- Simplices in $S_{x}^{n}$ which intersect $F$ transversally, and

- simplices in $S_{x}^{n}$ which have a vertex in $F$.

$\mu^{+}$vanishes on the second set, since it is determined on $S_{\text {cusp }}^{n}$ and the closed totally geodesic hypersurface $F$ can not have cusps. Thus, we obtain

$$
\mu^{+}\left(S_{x}^{n}\right)=\mu^{+}\left(S_{F}^{n} \cap S_{x}^{n}\right) \leq \mu^{+}\left(S_{F}^{n}\right),
$$

i.e., it suffices to show that $\mu^{+}\left(S_{x}^{n}\right)>0$.

For a measure $\mu^{+}$on $S S_{n}(M)$, let $\Phi_{X}\left(\mu^{+}\right)=\int_{S S_{n}(M)} \Phi_{x}(\sigma) d \mu^{+}(\sigma)$, where $\Phi_{x}(\sigma)$ is given in Definition 2.3. Weak-*-convergence implies $\Phi_{x}\left(\mu^{+}\right)$ $=\lim _{\epsilon \rightarrow 0} \Phi_{x}\left(c_{\epsilon}^{+}\right) \geq 1$.

On the other hand, $\Phi_{x}(\sigma)=0$ if $\sigma \notin S_{x}^{n}$, hence

$$
\Phi_{x}\left(\mu^{+}\right)=\int_{S_{x}^{n}} \Phi_{x}(\sigma) d \mu^{+}(\sigma) .
$$

If $\mu^{+}\left(S_{x}^{n}\right)=0$, then $\Phi_{x}\left(\mu^{+}\right)=\int_{S_{x}^{n}} \Phi_{x}(\sigma) d \mu^{+}(\sigma)=0$ (regardless whether $\Phi_{x}$ is bounded or not), giving a contradiction. Thus $\mu^{+}\left(S_{x}^{n}\right)>0$, implying $\mu^{+}\left(S_{F}^{n}\right)>0$.

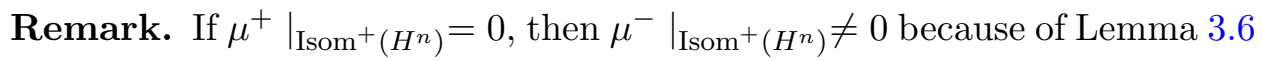
and Lemma 3.10, and we get with an analogous proof $\mu^{-}\left(S_{F}^{n}\right) \neq 0$.

\section{Acylindrical hyperbolic manifolds.}

In this chapter we extend Corollary 1 from [11] to manifolds with cusps.

If $\mathrm{M}$ is a hyperbolic manifold, define its convex core to be the smallest closed convex subset $C_{M}$ of $M$ such that the embedding $C_{M} \rightarrow M$ is a homotopy equivalence. $C_{M}$ is either contained in a geodesic codimension 1 submanifold, or it is a codimension 0 submanifold with boundary $\partial C_{M}$. (If $\operatorname{dim}(M)=3$, then $\partial C_{M}$ is, in general, a pleated surface (see [19]), i.e., 
is almost everywhere totally geodesic and is bent along a family of disjoint geodesics.) We say that $M$ has totally geodesic boundary if $C_{M}$ is homeomorphic to $\mathrm{M}$ and $\partial C_{M}$ is a nonempty totally geodesic submanifold of $M$. Note that we admit that $C_{M}$ may have cusps. If $M$ is an orientable geometrically finite hyperbolic 3 -manifold (with $C_{M}$ not contained in a geodesic codimension 1 submanifold), then $\partial C_{M}$ is homeomorphic to the union of all non-torus components of the topological boundary $\partial M$. This applies in particular to any hyperbolic 3-manifold $M$ with totally geodesic boundary $\partial C_{M}$.

Although hyperbolic structures of infinite volume are not necessarily rigid, it follows easily from Mostow's rigidity theorem that on a manifold of dimension $\geq 3$, there can be at most one hyperbolic metric $g_{0}$ admitting totally geodesic boundary, up to isometry. In particular, the volume of the convex core with respect to the metric $g_{0}$ is a topological invariant. Actually, it was shown in $[\mathbf{3}]$ that $g_{0}$ minimizes the volume of the convex core among all hyperbolic metrics on $\mathrm{M}$.

Lemma 6.1. Let $M$ be a compact 2-manifold with boundary $\partial M=\partial_{0} M \cup$ $\partial_{1} M$, such that $M-\partial_{0} M$ admits an incomplete hyperbolic metric of finite volume with $\partial_{1} M$ totally geodesic and the ends corresponding to $\partial_{0} M$ complete. Then

$$
\|M, \partial M\|=\frac{1}{V_{2}} \operatorname{Vol}(M) .
$$

Proof. It is well-known that any (possibly bounded) surface of nonpositive Euler characteristic satisfies $\|M, \partial M\|=-2 \chi(M)$. By the Gauß-Bonnetformula, this is the same as $\frac{1}{\pi} \mathrm{Vol}(M)=\frac{1}{V_{2}} \mathrm{Vol}(M)$.

Corollary 6.2. Let $n \geq 3$ and let $M$ be a compact $n$-manifold with boundary $\partial M=\partial_{0} M \cup \partial_{1} M$, such that $M-\partial_{0} M$ admits an incomplete hyperbolic metric of finite volume with $\partial_{1} M$ totally geodesic and the ends corresponding to $\partial_{0} M$ complete. Then,

$$
\|M, \partial M\|>\frac{1}{V_{n}} \operatorname{Vol}(M) .
$$

Proof. $\|M, \partial M\| \geq \frac{1}{V_{n}} \operatorname{Vol}(M)$ follows from the familiar argument that fundamental cycles can be straightened to invoke only simplices of volume smaller than $V_{n}$ or, equivalently, from the trivial inequality $\|D M\| \leq$ $2\|M, \partial M\|$.

Suppose we had equality $\|M, \partial M\|=\frac{1}{V_{n}} \operatorname{Vol}(M)$. Glue two differently oriented copies of $M$ via id $\left.\right|_{\partial M}$ to get $N=D M$. The incomplete metrics can be glued along the totally geodesic boundary and, hence, we have that $N$ is a complete hyperbolic manifold of finite volume $\operatorname{Vol}(N)=2 \operatorname{Vol}(M)$. A relative fundamental cycle for $\mathrm{M}$ of norm smaller than $\frac{1}{V_{n}} \operatorname{Vol}(M)+\frac{\epsilon}{2}$ fits together with its reflection to give a relative fundamental cycle $c_{\epsilon}$ on $N$ 
of $l^{1}$-norm smaller than $2 \frac{1}{V_{n}} \operatorname{Vol}(M)+\epsilon=\frac{1}{V_{n}} \operatorname{Vol}(N)+\epsilon$, but consisting of simplices which do not intersect transversally the totally geodesic surface $\partial M \subset N$, i.e., $c_{\epsilon}^{ \pm}\left(S_{\partial M}^{n}\right)=0$.

Recall that a representative $c_{\epsilon}$ of $[N, \partial N]$ was used in Chapter 3 to get a representative $\operatorname{Str}\left(\operatorname{exc}\left(r_{\epsilon *} c_{\epsilon}^{ \pm}\right)\right)$of $\left[\operatorname{int}(N), N_{[0, \epsilon]}\right]$ with (at most) the same $l^{1}$-norm. Here $r_{\epsilon}:(N, \partial N) \rightarrow\left(N_{[\epsilon, \infty]}, \partial N_{[\epsilon, \infty]}\right)$ was a homeomorphism, exc was the canonical inclusion, and Str means straightening. $c_{\epsilon}^{ \pm}\left(S_{\partial M}^{n}\right)=0 \mathrm{im}-$ plies $\operatorname{Str}\left(\operatorname{exc}\left(r_{\epsilon *} c_{\epsilon}^{ \pm}\right)\right)\left(S_{\partial M}^{n}\right)=0$, because $r_{\epsilon}$ can be choosen to be the identity in a neighborhood of the totally geodesic hypersurface $\partial M \subset N$ (which belongs to the thick part if $\epsilon$ is small enough), and because straightening in $N=D M$ preserves the set of simplices not intersecting transversally the totally geodesic surface $\partial M$.

By Lemma 3.2, we have some accumulation point $\mu$ of $\operatorname{Str}\left(\operatorname{exc}\left(r_{\epsilon *} c_{\epsilon}\right)\right)$ for a sequence of $\epsilon$ tending to zero. Similarly to Lemma 5.2, it is easy to see that $S_{\partial M}^{n}$ is open in $S S_{n}(N)$. Hence, we can apply Part (ii) of Lemma 2.2 to get $\mu^{+}\left(S_{\partial M}^{n}\right)=0$. But this contradicts Theorem 5.3.

\section{Theorem 6.3.}

(a) Let $n \geq 3$ and let $M_{i}, i=1,2$ be compact $n$-manifolds with boundaries $\partial M_{i}=\partial_{0} M_{i} \cup \partial_{1} M_{i}$, such that $M_{i}-\partial_{0} M_{i}$ admit incomplete hyperbolic metrics of finite volume with $\partial_{1} M_{i}$ totally geodesic and the ends corresponding to $\partial_{0} M_{i}$ complete. If $\partial_{1}^{\prime} M_{i} \subset \partial_{1} M_{i}$ are nonempty sets of connected components of $\partial_{1} M_{i}, f: \partial_{1}^{\prime} M_{1} \rightarrow \partial_{1}^{\prime} M_{2}$ is an orientationreversing isometry, and $M=M_{1} \cup_{f} M_{2}$, then

$$
\|M, \partial M\|<\left\|M_{1}, \partial M_{1}\right\|+\left\|M_{2}, \partial M_{2}\right\| .
$$

(b) Let $n \geq 3$ and let $M_{0}$ be a compact $n$-manifold with boundary $\partial M_{0}=$ $\partial_{0} M_{0} \cup \partial_{1} M_{0}$, such that $M_{0}-\partial_{0} M_{0}$ admits an incomplete hyperbolic metric of finite volume with $\partial_{1} M_{0}$ totally geodesic and the ends corresponding to $\partial_{0} M_{i}$ complete. If $\partial_{1}^{\prime} M_{0} \subset \partial_{1} M_{0}$ is a nonempty set of connected components of $\partial_{1} M_{0}$, and $f: \partial_{1}^{\prime} M_{0} \rightarrow \partial_{1}^{\prime} M_{0}$ is an orientationreversing isometry of $\partial_{1}^{\prime} M_{0}$ exchanging the connected components by pairs, then, letting $M=M_{0} / f$,

$$
\|M, \partial M\|<\left\|M_{0}, \partial M_{0}\right\| .
$$

Proof. (a) The incomplete hyperbolic metrics on $M_{1}$ and $M_{2}$ glue together to give a complete hyperbolic metric on $\mathrm{M}$ of volume $\operatorname{Vol}(M)=\operatorname{Vol}\left(M_{1}\right)+$ $\operatorname{Vol}\left(M_{2}\right)$. By the Gromov-Thurston theorem, we know that $\|M, \partial M\|=$ $\frac{1}{V_{n}} \operatorname{Vol}(M)$ and, by Corollary 6.2 , we have $\left\|M_{i}, \partial M_{i}\right\|>\frac{1}{V_{n}} \operatorname{Vol}\left(M_{i}\right)$. The claim follows.

The proof of (b) is similar.

If $\partial M_{i}$ has dimension $\geq 3$, any homeomorphism is homotopic to an isometry, by Mostow rigidity. We conclude: 
Corollary 6.4. Let $n \geq 4$, and let $M_{1}, M_{2}, M_{0}$ and $\partial_{1}^{\prime} M_{i}$ satisfy all assumptions of Theorem 6.3 .

If $f: \partial_{1}^{\prime} M_{1} \rightarrow \partial_{1}^{\prime} M_{2}$ is a homeomorphism, then

$$
\left\|M_{1} \cup_{f} M_{2}, \partial\left(M_{1} \cup_{f} M_{2}\right)\right\|<\left\|M_{1}, \partial M_{1}\right\|+\left\|M_{2}, \partial M_{2}\right\| .
$$

If $f: \partial_{1}^{\prime} M_{0} \rightarrow \partial_{1}^{\prime} M_{0}$ is an orientation-reversing homeomorphism of $\partial_{1}^{\prime} M_{0}$ exchanging the boundary components by pairs, then

$$
\left\|M_{0} / f, \partial\left(M_{0} / f\right)\right\|<\left\|M_{0}, \partial M_{0}\right\| \text {. }
$$

\section{Branching of laminations.}

We are going to extend results of [4] to manifolds with cusps (which are not Gieseking-like).

In this chapter, we always consider foliations/laminations of codimension 1.

For more background on the Gromov norm of foliations (and foliations in general), we refer to [4].

Definition 7.1. Let $M$ be a manifold, possibly with boundary, and $\mathcal{F}$ a lamination of M. Define

$$
\|M, \partial M\|_{\mathcal{F}}:=\inf \left\{\sum_{i=1}^{r}\left|a_{i}\right|: \sum_{i=1}^{r} a_{i} \sigma_{i} \in[M, \partial M], \sigma_{i} \text { transverse to } \mathcal{F}\right\} .
$$

Here, a simplex $\sigma$ is said to be transverse to the lamination $\mathcal{F}$, if the induced lamination $\left.\mathcal{F}\right|_{\sigma}$ is topologically conjugate to the subset of a foliation of $\sigma$ by level sets of an affine map $f: \sigma \rightarrow R$.

A typical example for non-transversality of a tetrahedron $\Delta$ to a lamination $\mathcal{F}$ is the following: Let $e_{1}, e_{2}, e_{3}$ be the three edges of a face $\tau \subset \Delta$. If $\left.\mathcal{F}\right|_{\tau}$ contains three lines which connect respectively $e_{1}$ to $e_{2}, e_{2}$ to $e_{3}$ and $e_{3}$ to $e_{1}$, then $\Delta$ can not be transverse to $\mathcal{F}$.

Remark. If $\mathcal{F}$ is not transverse to $\partial M$ nor contains $\partial M$ as a leaf, then $\|M, \partial M\|_{\mathcal{F}}=\infty$. Otherwise the foliated Gromov norm is finite. In the following, we will always assume that either $\mathcal{F}$ is transverse to $\partial M$ or that $\partial M$ is a leaf of $\mathcal{F}$.

7.1. Laminations with no or one-sided branching. Recall that a codimension 1 lamination $\mathcal{F}$ of an $n$-manifold $M$ is a decomposition of a closed subset $\lambda \subset M$ into codimension 1 submanifolds (leaves) so that $M$ is covered by charts of the form $I^{n-1} \times I$, the intersection of a leaf with a chart being of the form $I^{n-1} \times\{*\}$. A lamination $\mathcal{F}$ of a 3 -manifold $M$ with image $\lambda \subset M$ is called essential if no leaf is a sphere or a torus bounding a solid torus, $\overline{M-\lambda}$ is irreducible, and $\partial \overline{M-\lambda}$ is incompressible and end-incompressible in $\overline{M-\lambda}$, where the closure of $M-\lambda$ is taken w.r.t. any 
metric (see [8], Ch.1). E.g., if $M$ is a 3 -manifold and $\mathcal{F}$ a foliation without Reeb components, this is an essential lamination.

To motivate the following results about the relation between foliated Gromov norm and branching of laminations, we recall a notion from [8]. An order tree is a set $T$ together with a collection $S$ of linearly ordered segments $\sigma$, each having distinct least and greatest elements, $e(\sigma)$ and $i(\sigma)$, respectively, satisfying the following conditions:

- If $\sigma \in S$, then $-\sigma \in S$ (the set $\sigma$ with reversed order),

- any closed subset of $\sigma \in S$ belongs to $S$,

- any $v, w \in T$ can be joined by some $\sigma_{1}, \ldots, \sigma_{k} \in S$, i.e., $v=i\left(\sigma_{1}\right)$, $e\left(\sigma_{j}\right)=i\left(\sigma_{j+1}\right)$ and $w=e\left(\sigma_{k}\right)$,

- any cyclic word $\sigma_{0}, \ldots, \sigma_{k-1}$ with $e\left(\sigma_{j}\right)=i\left(\sigma_{j+1}\right)$ for all $j$ and $e\left(\sigma_{k-1}\right)$ $=i\left(\sigma_{0}\right)$ becomes trivial after subdividing the $\sigma_{i}$ 's and performing trivial cancellations,

- if $\sigma_{1} \cap \sigma_{2}=\left\{i\left(\sigma_{2}\right)\right\}=\left\{e\left(\sigma_{1}\right)\right\}$, then $\sigma_{1} \cup \sigma_{2} \in S$.

To a codimension 1 lamination $\mathcal{F}$ of $M$, one considers the pull-backlamination $\widetilde{\mathcal{F}}$ of $\widetilde{M}$ with image $\lambda \subset \widetilde{M}$, and one constructs $(T, S)$ as follows:

Elements of $T$ are either leaves of $\widetilde{\mathcal{F}}$ not contained in $\overline{\widetilde{M}}-\lambda$ or components of $\widetilde{M}-\lambda$. For each directed arc $\alpha$ intersecting leaves of $\widetilde{\mathcal{F}}$ transversally, having nonempty intersection with at least two leaves and being effective (no two points on the intersection with a leaf can be cancelled by an obvious homotopy), let $\sigma$ be the set of elements (of $T$ ) intersected by $\alpha$, with the inherited linear order.

According to [8], Prop. 6.10, $(T, S)$ is an order tree if $\mathcal{F}$ is an essential lamination.

$\mathcal{F}$ is then called $\mathbb{R}$-covered, one-sided branched, or two-sided branched according to whether the leaf space of $\widetilde{\mathcal{F}}$, considered as an order tree, is $\mathbb{R}$, branched in one direction, or branched in both directions.

Lemma 7.2. If $\mathcal{F}$ is an $\mathbb{R}$-covered or one-sided branched essential lamination on a 3-manifold $M$ such that $\left.\mathcal{F}\right|_{\partial M}$ is $\mathbb{R}$-covered, then

$$
\|M, \partial M\|=\|M, \partial M\|_{\mathcal{F}} .
$$

Proof. This is shown in Theorems 2.2.10 and 2.5.9 of [4], assuming that $M$ is closed. However, the proof works also for manifolds with boundary.

Indeed, since $\partial M$ is either transverse to $\mathcal{F}$ or is a leaf of $\mathcal{F}$, the straightening defined in Lemma 2.2.8 of [4], for chains with vertices on comparable leaves, preserves $C_{*}(\partial M)$. This implies, in particular, the claim for $\mathbb{R}$-covered foliations. In the case of one-sided branching (say in positive direction), the argument in 2.5.9 of [4] was then to isotope a chosen lift of the finite singular chain in $\widetilde{M}$ in the negative direction until its vertices are on comparable leaves. (This has to be done $\pi_{1} M$-equivariantly in the sense that the projection to $M$ stays a relative cycle.) If $\partial M$ is a leaf of $\mathcal{F}$, then 
one can leave all vertices on $\partial M$ fixed and only isotope the other vertices. If $\partial M$ is transversal to $\mathcal{F}$, the isotopy can clearly be performed in such a way that vertices on $\partial M$ (which already are on comparable leaves since $\left.\mathcal{F}\right|_{\partial M}$ is $\mathbb{R}$-covered) are isotoped inside $\partial M$.

Hence, in any case, the straightening maps $C_{*}(\partial M)$ to $C_{*}(\partial M)$ and, by the five lemma, it induces the identity map in relative homology. Thus, it maps relative fundamental cycles to relative fundamental cycles transversal to $\mathcal{F}$, not increasing the $l^{1}$-norm.

\subsection{Asymptotically separated laminations.}

Definition 7.3. Let $\operatorname{int}\left(M^{n}\right)$ be hyperbolic and let $\mathcal{F}$ be a lamination of $M$. Let $\left.\widetilde{\mathcal{F}}\right|_{\operatorname{int}(\widetilde{M})}$ be the pull-back lamination of $\mathbb{H}^{n} . \mathcal{F}$ is called asymptotically separated if, for some leaf $F \in \widetilde{\mathcal{F}}$, there are two geodesic $(n-1)$-planes on distinct sides of $F$.

We include a proof of the following lemma, implicit in [4], for lack of an explicit reference and because it might help to understand the idea behind Theorem 7.5.

Lemma 7.4. If $\mathcal{F}$ is an asymptotically separated lamination of a finitevolume hyperbolic manifold $M=\Gamma \backslash \mathbb{H}^{n}$, then $\mathcal{F}$ is two-sided branched.

Proof. Let $F$ be a leaf of $\widetilde{\mathcal{F}}$ such that there exist geodesic $(n-1)$-planes on distinct sides of $F$. These two planes cut out two half-spaces $U_{1}$ and $U_{2}$ on distinct sides of $F \subset \mathbb{H}^{n}$. Let $H$ be the complement of $U_{1}$ and let $H_{1}$ and $\mathrm{H}_{2}$ be disjoint half-spaces in $U_{2}$. Note that $F \subset H$.

If $\Gamma \backslash \mathbb{H}^{n}$ has finite volume, then it is well-known that the $\Gamma$-orbits on the space of pairs of distinct points in $\partial_{\infty} \mathbb{H}^{n}$ are dense.

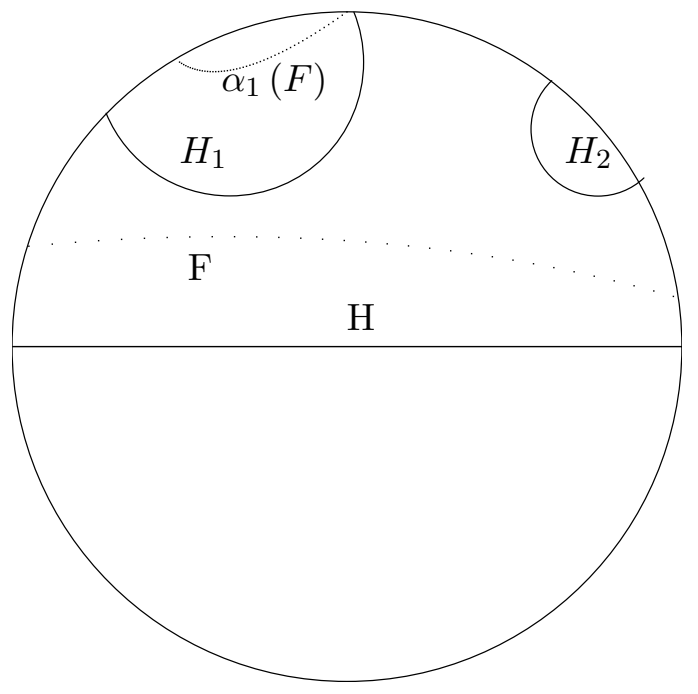


In particular, fixing some arbitrary $\gamma \in \Gamma$ with fixed points $p_{1}, p_{2}$, one finds conjugates of $\gamma$ in $\Gamma$, such that their fixed points come arbitrarily close to two given points $q_{1} \neq q_{2}$ in $\partial_{\infty} \mathbb{H}^{n}$. (Namely, conjugate with elements of $\Gamma$ which map $p_{1}$ close to $q_{1}$ and $p_{2}$ close to $q_{2}$.)

It follows that, in a finite-covolume subgroup $\Gamma \subset \operatorname{Isom}^{+}\left(\mathbb{H}^{n}\right)$, to any given disk $D \subset \partial_{\infty} \mathbb{H}^{n}$, one finds loxodromic isometries with both fixed points in this disk. Let $\alpha_{1}$ resp. $\alpha_{2}$ be such loxodromic isometries with both fixed points in $\partial_{\infty} H_{1}$ resp. both fixed points in $\partial_{\infty} H_{2}$. Loxodromic isometries map any set in the complement of a neighborhood of the repelling fixed point, after sufficiently many iterations, inside any neighborhood of the attracting fixed point. Hence, replacing $\alpha_{1}$ and $\alpha_{2}$ by sufficiently large powers, we get that $\alpha_{1}(F) \subset H_{1}$ and $\alpha_{2}(F) \subset H_{2}$.

Since $\widetilde{\mathcal{F}}$ is $\Gamma$-invariant, we have found incomparable leaves $\alpha_{1}(F)$ and $\alpha_{2}(F)$ above $F$ and, by analogous arguments, we also get incomparable leaves below $F$.

Remark. A conjecture of Fenley would imply that a foliation of a finitevolume hyperbolic 3-manifold int $(M)$ is two-sided branched if and only if it is asymptotically separated, see the discussion in Chapter 2.5. of [4]. Namely, Calegari proves that a two-sided branched foliation (on an arbitrary hyperbolic manifold) either is asymptotically separated or the leaves have as limit sets all of $\partial_{\infty} \mathbb{H}^{3}$. On the other hand, Fenley conjectures that for foliations of finite-volume hyperbolic manifolds (which are transversal to the boundary $\partial M)$, the limit set of a leaf can be all of $\partial_{\infty} \mathbb{H}^{3}$ only if $\mathcal{F}$ is $\mathbb{R}$-covered.

The following Theorem 7.5 extends Theorem 2.4.5 in [4] to the cusped case.

Theorem 7.5. If the interior of $M$ is a hyperbolic $n$-manifold of finite volume which is not Gieseking-like, $n \geq 3$, and if $\mathcal{F}$ is an asymptotically separated lamination, then

$$
\|M, \partial M\|<\|M, \partial M\|_{\mathcal{F}} .
$$

Proof. We want to give an outline of the proof. We will show that there exist three half-spaces $D_{0}, D_{1}, D_{2}$ such that the following holds: Whenever a straight simplex has at least one vertex in each of $D_{0}, D_{1}, D_{2}$, it can not be transverse to $\mathcal{F}$. Assuming $\|M, \partial M\|_{\mathcal{F}}=\|M, \partial M\|$, we would have an efficient fundamental cycle $\mu$ which actually comes from a sequence of fundamental cycles transverse to $\mathcal{F}$. If $M$ is closed, one gets easily that $\mu^{ \pm}$ have to vanish on the set of those ideal simplices with at least one vertex in each of $\partial_{\infty} D_{0}, \partial_{\infty} D_{1}, \partial_{\infty} D_{2}$. If $M$ has cusps, we still get the slightly weaker statement that $\mu^{ \pm}$have to vanish on the set of those ideal simplices with at least one vertex in each of $\partial_{\infty} D_{0}-P, \partial_{\infty} D_{1}-P, \partial_{\infty} D_{2}-P$, where $P$ is the 
set of parabolic fixed points of $\Gamma$. We can then use our knowledge of $\mu$ to derive a contradiction.

Let $F$ be a leaf which has the property in the definition of "asymptotically separated", i.e., there are planes, and hence half-spaces $U_{1}$ and $U_{2}$, in disjoint components of $\mathbb{H}^{n}-F$. We choose in $U_{2}$ two smaller disjoint half-spaces $H_{1}$ and $H_{2}$. Like in the proof of Lemma 7.4, one finds loxodromic isometries $\alpha_{1} \in \Gamma$ with both fixed points in $H_{1}$ and $\alpha_{2} \in \Gamma$ with both fixed points in $H_{2}$. Replacing, if necessary, $\alpha_{1}$ and $\alpha_{2}$ by sufficiently large powers, we arrange that $\alpha_{1}\left(U_{1}\right) \subset H_{1}$ and $\alpha_{2}\left(U_{1}\right) \subset H_{2}$, and that $F, \alpha_{1}(F), \alpha_{2}(F)$ are disjoint. Letting $D_{0}=U_{2}, D_{1}=\alpha_{1}\left(U_{1}\right)$, and, $D_{2}=\alpha_{2}\left(U_{1}\right)$, the remark after Definition 7.1 tells us that there is no tetrahedron transverse to $\widetilde{\mathcal{F}}$ with one vertex in each of $D_{0}, D_{1}$ and $D_{2}$.

For the convenience of the reader, we first explain the proof for closed manifolds. Assume that we have straight fundamental cycles $c_{\epsilon}$, transverse to $\mathcal{F}$, with $\left\|c_{\epsilon}\right\|<\|M\|+\frac{\epsilon}{V_{n}}$, and that $\mu$ is the weak-*-limit of $c_{\epsilon}$. Denoting by $S_{D_{0}, D_{1}, D_{2}}$ the open set of straight (possibly ideal) simplices with one vertex in each of $D_{0}, D_{1}$ and $D_{2}$, we have just seen that transversality to $\mathcal{F}$ implies $c_{\epsilon}^{ \pm}\left(S_{D_{0}, D_{1}, D_{2}}\right)=0$. This implies $\mu^{ \pm}\left(S_{D_{0}, D_{1}, D_{2}}\right)=0$, contradicting the fact that $\mu^{+}$is the Haar measure. (A similar argument was given by Calegari in 2.4 .5 of $[4]$.)

Now we are going to consider hyperbolic manifolds of finite volume. Let $P \subset \partial_{\infty} \mathbb{H}^{n}$ be the parabolic fixed points of $\Gamma$, and $H_{\epsilon}=p^{-1}\left(M_{[0, \epsilon]}\right) \subset$ $\mathbb{H}^{n}$ the preimage of the $\epsilon$-thin part. It is the union of horoballs centered at the points of $P$. For $\delta$ sufficiently small, $D_{0}-\overline{H_{\delta}}, D_{1}-\overline{H_{\delta}}$ and $D_{2}-\overline{H_{\delta}}$ are nonempty. Fix such a $\delta$. Let

$$
\begin{aligned}
S_{D_{0}, D_{1}, D_{2}}=\{\text { simplices having vertices } & v_{0} \in D_{0}-\overline{H_{\delta}}, \\
& \left.v_{1} \in D_{1}-\overline{H_{\delta}}, v_{2} \in D_{2}-\overline{H_{\delta}}\right\},
\end{aligned}
$$

where we admit ideal simplices.

We have seen that simplices in $S_{D_{0}, D_{1}, D_{2}}$ are not transversal to $\widetilde{\mathcal{F}}$. Moreover, we define

$$
\operatorname{Str}\left(S_{D_{0}, D_{1}, D_{2}}\right):=\left\{\operatorname{Str}(\sigma): \sigma \in S_{D_{0}, D_{1}, D_{2}}\right\}
$$

and

$\mathbb{U}:=\left\{\right.$ pos. or. regular ideal simplices $\left(v_{0}, \ldots, v_{n}\right): v_{i} \in \partial_{\infty} D_{i}-P$

$$
\text { for } i=0,1,2\} \text {. }
$$

Now suppose we had the equality $\|M, \partial M\|=\|M, \partial M\|_{\mathcal{F}}$. We will stick to the notations of Chapter 3. Take some transverse relative fundamental 
cycle $c_{\epsilon}$ of norm smaller than $\|M, \partial M\|+\frac{\epsilon}{V_{n}}$ and make it, via the homeomorphism $r_{\epsilon}:(M, \partial M) \rightarrow\left(M_{[\epsilon, \infty]}, \partial M_{[\epsilon, \infty]}\right)$, to a relative fundamental cycle $r_{\epsilon *}\left(c_{\epsilon}\right)$ of the $\epsilon$-thick part, which is transverse to the foliation $r_{\epsilon}(\mathcal{F})$. We may arrange $r_{\epsilon}$ to be the identity on the $\epsilon^{\prime}$-thick part for $\epsilon^{\prime}$ slightly larger than $\epsilon$. Then, the lift of $r_{\epsilon *}\left(c_{\epsilon}\right)$ to $\mathbb{H}^{n}$ is transverse to $\widetilde{\mathcal{F}}$ outside $H_{\epsilon^{\prime}}$. By choosing $\epsilon$ and $\epsilon^{\prime}$ sufficiently small, one may make this exceptional set $H_{\epsilon^{\prime}}$ as small as one wishes.

Decompose $S_{D_{0}, D_{1}, D_{2}}$ as a countable union $S_{D_{0}, D_{1}, D_{2}}=\cup_{i=1}^{\infty} V_{i}$, where $V_{i} \subset S_{D_{0}, D_{1}, D_{2}}$ is the open subset of (possibly ideal) positively oriented simplices $\sigma \in S_{D_{0}, D_{1}, D_{2}}$ satisfying $\sigma \cap H_{\frac{1}{i}}=\emptyset$. (The union is all of $S_{D_{0}, D_{1}, D_{2}}$ because any ideal or non-ideal simplex with vertices outside $H_{\delta}$ must remain outside some $H_{\frac{1}{i}}$ for sufficiently large $i$.) Let $W_{i}=\operatorname{Str}\left(V_{i}\right)=$ $\left\{\operatorname{str}(\sigma): \sigma \in V_{i}\right\}$. For $\epsilon$ sufficiently small (such that we can choose $\epsilon^{\prime}<\frac{1}{i}$ ), we have $r_{\epsilon *}\left(c_{\epsilon}^{ \pm}\right)\left(V_{i}\right)=0$, since $r_{\epsilon *}\left(c_{\epsilon}\right)$ is transverse to $\mathcal{F}$ outside $H_{\frac{1}{i}}$ and $V_{i}$ consists of simplices which do not intersect $H_{\frac{1}{i}}$ and which are not transverse to $\mathcal{F}$. As a consequence, $\operatorname{Str}\left(\operatorname{exc}\left(r_{\epsilon *}\left(c_{\epsilon}\right)\right)\right)\left(W_{i}\right)=0$. If $\mu$ is the weak-* limit of the sequence $\operatorname{Str}\left(\operatorname{exc}\left(r_{\epsilon *}\left(c_{\epsilon}\right)\right)\right)$ with $\epsilon \rightarrow 0$, we get $\mu^{ \pm}\left(W_{i}\right)=0$ by openness of $W_{i}$ and Part (ii) of Lemma 2.2.

$W=\operatorname{Str}\left(S_{D_{0}, D_{1}, D_{2}}\right)=\left\{\operatorname{Str}(\sigma): \sigma \in S_{D_{0}, D_{1}, D_{2}}\right\}$ is a countable increasing union $W=\cup_{i=1}^{\infty} W_{i}$. Hence $\mu^{ \pm}(W)=0$. $\mathbb{U} \subset W$ implies

$$
\mu^{ \pm}(\mathbb{U})=0 .
$$

On the other hand, $\mathbb{U}$ has nontrivial Haar measure. Indeed, $\operatorname{Isom}^{+}\left(\mathbb{H}^{n}\right)$ corresponds to ordered $n$-tuples of points in $\partial_{\infty} \mathbb{H}^{n}$, because any such ordered $n$-tuple is the set of first $n$ vertices for some unique positively oriented ordered regular ideal simplex. Hence, the set of positive regular ideal simplices, with $v_{i} \in \partial_{\infty} D_{i}$ for $i=0,1,2$, corresponds to an open set of positive Haar measure in $\operatorname{Isom}^{+}\left(\mathbb{H}^{n}\right)$. Clearly, a discrete subgroup of Isom ${ }^{+}\left(\mathbb{H}^{n}\right)$ has a countable number of parabolic fixed points. Thus, $\mathbb{U}$ has positive Haar measure.

Recall the notation from Chapter 4: $v \in \partial_{\infty} \mathbb{H}^{n}$ is an arbitrary vertex of the reference simplex $\Delta_{0}$ and $\beta_{v}(g)$ is the ergodic component of $g \in$ $\Gamma \backslash \operatorname{Isom}^{+}\left(\mathbb{H}^{n}\right)$ with respect to the $T_{v}$-action. We define

$$
H_{v}=\left\{g \in \Gamma \backslash G: \beta_{v}(g)=\text { Haar }\right\} .
$$

Haar $(\mathbb{U}) \neq 0$ implies $\mu^{ \pm}\left(H_{v}\right)=0$. Indeed, from Lemma 4.2 and Lemma 4.3 we know that the complement of $H_{v}$ in the set of regular ideal simplices is the set of simplices $g \Delta_{0}$ with the vertex $g(v)$ in a parabolic fixed point of $\Gamma$. $\Gamma$ has a countable number of parabolic fixed points and, therefore, this complement is a set of trivial Haar measure. Thus,

$$
\operatorname{Haar}\left(\mathbb{U} \cap H_{v}\right)=\operatorname{Haar}(\mathbb{U})>0
$$


and we apply the ergodic decomposition from 2.2 to get

$$
0=\mu^{ \pm}\left(\mathbb{U} \cap H_{v}\right)=\operatorname{Haar}\left(\mathbb{U} \cap H_{v}\right) \mu^{ \pm}\left(H_{v}\right)
$$

which implies

$$
\mu^{ \pm}\left(H_{v}\right)=0
$$

This discussion applies to all vertices $v_{i}$ of the reference simplex $\Delta_{0}$. By Lemma 5.5, we can conclude that $\mu^{ \pm}$are determined on $S_{\text {cusp }}^{n}$.

In particular, since $\mu \neq 0$, there necessarily are regular simplices with all vertices in parabolic fixed points. By Lemma 3.10, $\mu$ is invariant up to sign under the right-hand action of the regular ideal reflection group $R$ defined in Section 3.2. Hence, there must even be an $R$-invariant family of regular ideal simplices with vertices in parabolic fixed points. This is only possible in dimension 3 and, after conjugating with an isometry, $Q(\omega) \cup\{\infty\}$ must be parabolic fixed points of $\Gamma$.

A surface $F$ in a 3 -manifold $M$ is called a virtual fiber if there is some finite cover $p: \bar{M} \rightarrow M$ and some fibration $\bar{F} \rightarrow \bar{M} \rightarrow S^{1}$ with $\bar{F}$ isotopic to $p^{-1}(F)$.

A theorem of Thurston and Bonahon asserts that a properly embedded compact $\pi_{1}$-injective surface in a finite-volume hyperbolic 3 -manifold is either quasigeodesic or a virtual fiber. Since quasigeodesic surfaces are asymptotically separated, one gets analogously to [4], Theorem 4.1.4:

Corollary 7.6. If the interior of $M$ is a hyperbolic 3-manifold of finite volume which is not Gieseking-like and $F \subset M$ is a properly embedded compact $\pi_{1}$-injective surface, then $F$ is a virtual fiber if and only if $\|M, \partial M\|_{\mathcal{F}}=$ $\|M, \partial M\|$.

Acknowledgements. I would like to thank Michel Boileau and Bernhard Leeb for discussions about the content of this paper.

\section{References}

[1] W. Ballmann, M. Gromov and V. Schroeder, Manifolds of Nonpositive Curvature, Birkhäuser, Verlag, 1985, MR 87h:53050, Zbl 0591.53001.

[2] R. Benedetti and C. Petronio, Lectures on Hyperbolic Geometry, Universitext, Springer-Verlag, Berlin, 1992, MR 94e:57015, Zbl 0768.51018.

[3] F. Bonahon, A Schlaefli-type formula for the convex core of hyperbolic 3-manifolds, J. Differential Geom., 50 (1998), 25-58, MR 2000j:57049, Zbl 0944.53021.

[4] D. Calegari, The Gromov norm and foliations, Geom. Funct. Anal., 10 (2000), 14231447, MR 2002c:57026a, Zbl 0974.57015, Zbl 0977.57035; Publisher's erratum, Geom. Funct. Anal., 11 (2001), 199, MR 2002c:57026b, Zbl 0990.57006.

[5] S. Dani, Invariant measures of horospherical flows on noncompact homogeneous spaces, Invent. Math., 47 (1978), 101-138, MR 58 \#28260, Zbl 0368.28021. 
[6] - On uniformly distributed orbits of certain horocycle flows, Ergod. Th. Dynam. Sys., 2 (1982), 139-158, MR 84g:58068, Zbl 0504.22006.

[7] S. Fenley, Limit sets of foliations in hyperbolic 3-manifolds, Topology, 37 (1998), 875-894, MR 2000e:57029, Zbl 0896.57018.

[8] D. Gabai and U. Oertel, Essential laminations in 3-manifolds, Ann. of Math. (2), 130 (1989), 41-73, MR 90h:57012, Zbl 0685.57007.

[9] M. Gromov, Volume and bounded cohomology, Publ. Math. IHES, 56 (1982), 5-99, MR 84h:53053, Zbl 0516.53046.

[10] U. Haagerup and H. Munkholm, Simplices of maximal volume in hyperbolic $n$-space, Acta Math., 147 (1981), 1-11, MR 82j:53116, Zbl 0493.51016.

[11] D. Jungreis, Chains that realize the Gromov invariant of hyperbolic manifolds, Ergod. Th. Dynam. Sys., 17 (1997), 643-648, MR 98c:57013, Zbl 0882.57009.

[12] Th. Kuessner, Multicomplexes, bounded cohomology and additivity of simplicial volume. Preprint, math.AT/0109057.

[13] D.D. Long and A.W. Reid, Pseudomodular surfaces, J. Reine Angew. Math., 552 (2002), 77-100, CMP 1940433.

[14] C.C. Moore, Ergodicity of flows on homogeneous spaces, Amer. J. Math., 88 (1966), 154-178, MR 33 \#1409, Zbl 0148.37902.

[15] J.-P. Otal, Le theoreme d'hyperbolisation pour les varietes fibrees de dimension 3, Asterisque, 235 (1996), MR 97e:57013, Zbl 0855.57003.

[16] N. Peyerimhoff, Simplices of maximal volume or minimal total edge length in hyperbolic space, J. London Math. Soc., 66 (2002), 758-768, CMP 1934304.

[17] M. Ratner, Raghunathan's topological conjecture and distributions of unipotent flows, Duke Math. J., 63 (1991), 235-280, MR 93f:22012, Zbl 0733.22007.

[18] T. Soma, Volume of hyperbolic 3-manifolds with iterated pseudo-Anosov amalgamations, Geom. Dedic., 90 (2002), 183-200, MR 2003a:57032, Zbl 0996.57009.

[19] W. Thurston, Three-dimensional Geometry and Topology, Vol. 1, Edited by Silvio Levy. Princeton Mathematical Series, 35, Princeton University Press, Princeton, NJ, 1997, MR 97m:57016, Zbl 0873.57001.

[20] , Hyperbolic geometry and 3-manifolds, Low-dimensional topology, Proc. Conf. Bangor, Lond. Math. Soc. Lect. Note Ser., 48 (1982), 9-25, MR 83j:57006, Zbl 0483.57007.

[21] V.S. Varadarajan, Groups of automorphisms of Borel spaces, Trans. Amer. Math. Soc., 109 (1963), 191-219, MR 28 \#3139, Zbl 0192.14203.

[22] A. Zastrow, On the (non)-coincidence of Milnor-Thurston homology theory with singular homology theory, Pacific J. Math., 186 (1998), 369-396, MR 2000a:55008, Zbl 0933.55008 .

Received April 19, 2002 and revised December 14, 2002.

Mathematische Fakultät

LMU MÜNCHEN, 80333 MÜNCHEN

Germany

E-mail address: kuessner@mathematik.uni-muenchen.de 